\title{
HERSCHEL FAR-INFRARED AND SUBMILLIMETER PHOTOMETRY FOR THE KINGFISH SAMPLE OF NEARBY GALAXIES
}

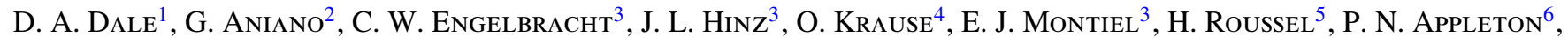 \\ L. Armus ${ }^{7}$, P. Beirão ${ }^{7}$, A. D. Bolatto ${ }^{8}$, B. R. Brandl ${ }^{9}$, D. CAlzetti $^{10}$, A. F. Crocker ${ }^{10}$, K. V. Croxall ${ }^{11}$, B. T. Draine ${ }^{2}$,

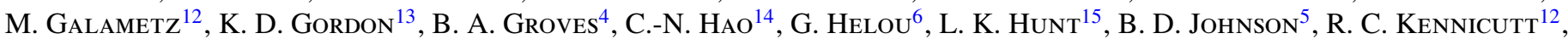 \\ J. Koda ${ }^{16}$, A. K. Leroy ${ }^{17}$, Y. Li ${ }^{10}$, S. E. MeidT ${ }^{4}$, A. E. Miller ${ }^{1}$, E. J. Murphy ${ }^{7}$, N. Rahman ${ }^{8}$, H.-W. Rix ${ }^{4}$, K. M. SandSTrom ${ }^{4}$, \\ M. Sauvage ${ }^{18}$, E. Schinnerer ${ }^{4}$, R. A. Skibba ${ }^{3}$, J.-D. T. Smith ${ }^{11}$, F. S. TAbatabaei ${ }^{4}$, F. Walter ${ }^{4}$, C. D. Wilson ${ }^{19}$, \\ M. G. Wolfire ${ }^{8}$, AND S. ZibetTi ${ }^{20}$ \\ ${ }^{1}$ Department of Physics \& Astronomy, University of Wyoming, Laramie, WY 82071, USA; ddale@ uwyo.edu \\ ${ }^{2}$ Department of Astrophysical Sciences, Princeton University, Princeton, NJ 08544, USA \\ ${ }^{3}$ Steward Observatory, University of Arizona, Tucson, AZ 85721, USA \\ ${ }^{4}$ Max-Planck-Institut für Astronomie, Königstuhl 17, D-69117 Heidelberg, Germany \\ ${ }^{5}$ Institut d'Astrophysique de Paris, UMR7095 CNRS, Université Pierre \& Marie Curie, 98 bis Boulevard Arago, 75014 Paris, France \\ ${ }^{6}$ NASA Herschel Science Center, IPAC, California Institute of Technology, Pasadena, CA 91125, USA \\ ${ }^{7}$ Spitzer Science Center, California Institute of Technology, MC 314-6, Pasadena, CA 91125, USA \\ ${ }^{8}$ Department of Astronomy, University of Maryland, College Park, MD 20742, USA \\ ${ }^{9}$ Leiden Observatory, Leiden University, P.O. Box 9513, 2300 RA Leiden, The Netherlands \\ ${ }^{10}$ Department of Astronomy, University of Massachusetts, Amherst, MA 01003, USA \\ ${ }^{11}$ Department of Physics and Astronomy, University of Toledo, Toledo, OH 43606, USA \\ 12 Institute of Astronomy, University of Cambridge, Madingley Road, Cambridge CB3 OHA, UK \\ ${ }^{13}$ Space Telescope Science Institute, 3700 San Martin Drive, Baltimore, MD 21218, USA \\ ${ }^{14}$ Tianjin Astrophysics Center, Tianjin Normal University, Tianjin 300387, China \\ ${ }^{15}$ INAF-Osservatorio Astrofisico di Arcetri, Largo E. Fermi 5, 50125 Firenze, Italy \\ ${ }^{16}$ Department of Physics and Astronomy, SUNY Stony Brook, Stony Brook, NY 11794-3800, USA \\ ${ }^{17}$ National Radio Astronomy Observatory, 520 Edgemont Road, Charlottesville, VA 22903, USA \\ ${ }^{18}$ CEA/DSM/DAPNIA/Service d'Astrophysique, UMR AIM, CE Saclay, 91191 Gif sur Yvette Cedex, France \\ ${ }^{19}$ Department of Physics \& Astronomy, McMaster University, Hamilton, Ontario L8S 4M1, Canada \\ ${ }^{20}$ Dark Cosmology Centre, Niels Bohr Institute, University of Copenhagen, Juliane Maries Vej 30, DK-2100 Copenhagen, Denmark \\ Received 2011 October 25; accepted 2011 December 4; published 2012 January 4
}

\begin{abstract}
New far-infrared and submillimeter photometry from the Herschel Space Observatory is presented for 61 nearby galaxies from the Key Insights on Nearby Galaxies: A Far-Infrared Survey with Herschel (KINGFISH) sample. The spatially integrated fluxes are largely consistent with expectations based on Spitzer far-infrared photometry and extrapolations to longer wavelengths using popular dust emission models. Dwarf irregular galaxies are notable exceptions, as already noted by other authors, as their $500 \mu \mathrm{m}$ emission shows evidence for a submillimeter excess. In addition, the fraction of dust heating attributed to intense radiation fields associated with photodissociation regions is found to be $(21 \pm 4) \%$ larger when Herschel data are included in the analysis. Dust masses obtained from the dust emission models of Draine \& Li are found to be on average nearly a factor of two higher than those based on single-temperature modified blackbodies, as single blackbody curves do not capture the full range of dust temperatures inherent to any galaxy. The discrepancy is largest for galaxies exhibiting the coolest far-infrared colors.
\end{abstract}

Key words: galaxies: ISM - infrared: ISM - ISM: general

Online-only material: color figures

\section{INTRODUCTION}

The amount of dust contained within a galaxy reflects the integrated chemical enrichment of the interstellar medium (ISM) through accumulated episodes of star formation and any merger/accretion events, coupled with the galaxy's history of dust grain formation and destruction. Thus, the total amount of dust observed within a galaxy can be tied to its star formation history. From an observational viewpoint, estimating a galaxy's dust mass depends critically on far-infrared/submillimeter ${ }^{21}$ photometry and the inferred distribution of dust grain temperatures; long-wavelength data are crucial for probing cool 15-20 K dust with any accuracy, and dust emitting at this temperature range makes up the bulk of the dust mass in a typical star-forming

21 "Far-infrared" and "submillimeter" are defined here as $40 \leqslant \lambda \leqslant 300$ and $300 \leqslant \lambda \leqslant 1000 \mu \mathrm{m}$, respectively. galaxy (Dunne \& Eales 2001). Recent observational efforts have shown evidence for excess emission at submillimeter wavelengths in galaxies, particularly in low-metallicity systems such as the Small Magellanic Cloud and other dwarf galaxies (e.g., Bolatto et al. 2000; Galliano et al. 2005, 2011; Bot et al. 2010; Gordon et al. 2010; Ade et al. 2011a, 2011b; Galametz et al. 2011). If the excess emission is interpreted as evidence for particularly cold dust (e.g., $T \lesssim 10 \mathrm{~K}$ ), substantial amounts must be present in order to produce the observed emission, and the resulting dust-to-gas mass ratios inferred from these observations are significantly higher than what would be expected based on a galaxy's metallicity (see, e.g., Galametz et al. 2009; Meixner et al. 2010; Ade et al. 2011a). Alternative explanations for the excess emission include resonances due to impurities in the dust or a modified dust emissivity at submillimeter wavelengths, e.g., changes with environment in dust grain size and/or composition (see Lisenfeld et al. 2002; Aguirre et al. 2003; Dupac et al. 
2003; Meny et al. 2007; Galametz et al. 2009; Meixner et al. 2010, and references therein).

Understanding the physical origin of the submillimeter emission in nearby galaxies obviously requires sensitive submillimeter data. However, it has been difficult to obtain reliable submillimeter photometry. The opacity of the atmosphere limits ground-based observations to a few partially transparent submillimeter spectral windows, and previous space-based missions such as COBE, IRAS, ISO, Spitzer, and AKARI lacked the sensitive instrumentation and the requisite angular resolution for resolved studies of nearby galaxies at these wavelengths. The Herschel Space Observatory (Pilbratt et al. 2010) allows an unprecedented look into the long-wavelength emission from galaxies. Herschel provides impressive angular resolution in the far-infrared/submillimeter wavelength range and unparalleled sensitivity to low surface brightness emission at 250, 350, and $500 \mu \mathrm{m}$, wavelengths that bridge an important spectral gap between space-based galaxy surveys carried out at 160-170 $\mu \mathrm{m}$ (ISO, Spitzer, AKARI) and those from the ground at $850-870 \mu \mathrm{m}$; there are precious few detections of galaxies at 350 or $450 \mu \mathrm{m}$ of James Clerk Maxwell Telescope/SCUBA or Caltech Submillimeter Observatory/SHARC heritage (e.g., Benford et al. 1999; Dunne \& Eales 2001; Kovács 2006). Filling in this gap in wavelength coverage is particularly important for the coldest galaxies in the local universe in which the dust emission peaks at $\sim 150 \mu \mathrm{m}$ or longer wavelengths.

In this effort we present Herschel PACS (Poglitsch et al. 2010) and SPIRE (Griffin et al. 2010) broadband photometry for the 61 galaxies in KINGFISH (Key Insights on Nearby Galaxies: A Far-Infrared Survey with Herschel). The flux densities provided here are spatially integrated or "global" values, and thus they do not directly take advantage of one of the key features of Herschel data: angular resolution. However, the sharpness of the imaging comes into play by enabling superior identification and removal of contamination from neighboring and background galaxies. While this study of global flux densities only indirectly profits from Herschel's superior resolution, other studies investigate the spatially resolved properties of KINGFISH galaxies. For example, Walter et al. (2011), G. Aniano et al. (2012, in preparation), and K. D. Gordon et al. (2012a, in preparation) explore how the infrared spectral energy distributions vary with location in KINGFISH targets; M. Galametz et al. (2012, in preparation), J. Hinz et al. (2012, in preparation), and L. Hunt et al. (2012, in preparation) study cold dust emission in the outer disks; Y. Li et al. (2012, in preparation) and A. Crocker et al. (2012, in preparation) characterize the infrared properties of $\mathrm{H}$ II regions, etc.

The global flux densities presented here are used to see how well the far-infrared/submillimeter emission in nearby galaxies can be modeled using the theoretical spectral energy distribution curves of Draine \& Li (2007), curves that are based on the dust emission properties of the Milky Way. We also quantify whether the submillimeter data provide new insight into galaxy spectral energy distributions or whether their values are expected based on extrapolations from shorter wavelength data. Finally, we search for evidence for a global submillimeter excess in KINGFISH galaxies, explore the unique characteristics of the low-metallicity systems in the sample, and contrast the dust masses found through Draine \& Li (2007) fits with those from the common approach of fitting single-temperature modified blackbodies.

Section 2 describes the sample and Section 3 reviews the observations as well as the data processing and aperture photometry procedures that have been adopted here for KINGFISH imaging. In Section 4, the spatially integrated photometry data are presented in addition to the results of fits to the observed spectral energy distributions, and Section 5 provides a summary of our findings.

\section{SAMPLE}

The KINGFISH sample of galaxies draws from the Spitzer Infrared Nearby Galaxies Survey (SINGS; see Kennicutt et al. 2003); 57 SINGS galaxies are in the KINGFISH sample, plus NGC 2146, NGC 3077, NGC 5457, and IC 342. The 61 galaxies were selected to span wide ranges in luminosity, optical/infrared ratio, and morphology (Kennicutt et al. 2012). The luminosity ranges over a factor of $10^{4}$ (but all are sub-luminous infrared galaxies, or nearly so in the case of NGC 2146, with $L_{\mathrm{IR}}<$ $10^{11} L_{\odot}$ ), the range of optical/infrared ratios covers a factor of $10^{3}$, and all "normal" galaxy types are represented. There are several galaxies with nuclei that are clearly distinguished by Seyfert or LINER characteristics, but none of the galaxies have their global luminosity dominated by an active nucleus (Moustakas et al. 2010). The sample also spans representative ranges in metallicity, gas fraction, $\mathrm{HI} / \mathrm{H}_{2}$ ratio, star formation rate, and bar strength.

\section{OBSERVATIONS AND DATA PROCESSING}

The observational program and data processing procedures for KINGFISH are described in detail in Engelbracht et al. (2010), Sandstrom et al. (2010), and Kennicutt et al. (2012). A brief description is provided here. All 61 KINGFISH galaxies were imaged with PACS and SPIRE.

\subsection{PACS Data}

PACS imaging was obtained in scan mode, along two perpendicular axes for improved image reconstruction, at the medium scan speed of $20^{\prime \prime} \mathrm{s}^{-1}$. The $45^{\circ}$ orientation of the array with respect to the scan direction contributes to a more uniform spatial coverage. Two Astronomical Observation Requests (AORs) were carried out for joint 70 and $160 \mu \mathrm{m}$ imaging, and an additional two AORs were utilized for joint 100 and $160 \mu \mathrm{m}$ observations, resulting in a total of four AORs for $160 \mu \mathrm{m}$ imaging. Three or six repetitions were carried out for each AOR, depending on an individual galaxy's far-infrared surface brightness as gauged from Spitzer/MIPS data. The integrations achieved per pixel lead to approximate $1 \sigma$ surface brightness sensitivities of $\sigma_{\text {sky }} \sim 5,5$, and $2 \mathrm{MJy} \mathrm{sr}^{-1}$ at 70,100 , and $160 \mu \mathrm{m}$, respectively, for the fainter subset of galaxies and approximately $\sqrt{2}$ times larger for the brighter subset. The PACS calibration uncertainties are $\epsilon_{\mathrm{cal}, v} / f_{v} \sim 5 \%$, according to Version 2.3 (2011 June 8) of the PACS Observer's Manual.

The raw ("Level 0") data were processed using Version 5.0 of HIPE (Ott 2010). Besides the standard pipeline procedures, the conversion from Level 0 to Level 1 data included secondlevel deglitching and corrections for any offsets in the detector sub-matrices. Scanamorphos ${ }^{22}$ (Roussel 2011) Version 12.5 was used to process the Level 1 PACS scan map data. Its main task is to subtract the brightness drifts caused by the low-frequency noise (comprising both the thermal drifts of the telescope and detectors and the flicker noise of the individual bolometers), before projecting the data onto a changeable spatial grid. The algorithm employs minimal assumptions about the noise and the

\footnotetext{
$\overline{22}$ http://www2.iap.fr/users/roussel/herschel
} 


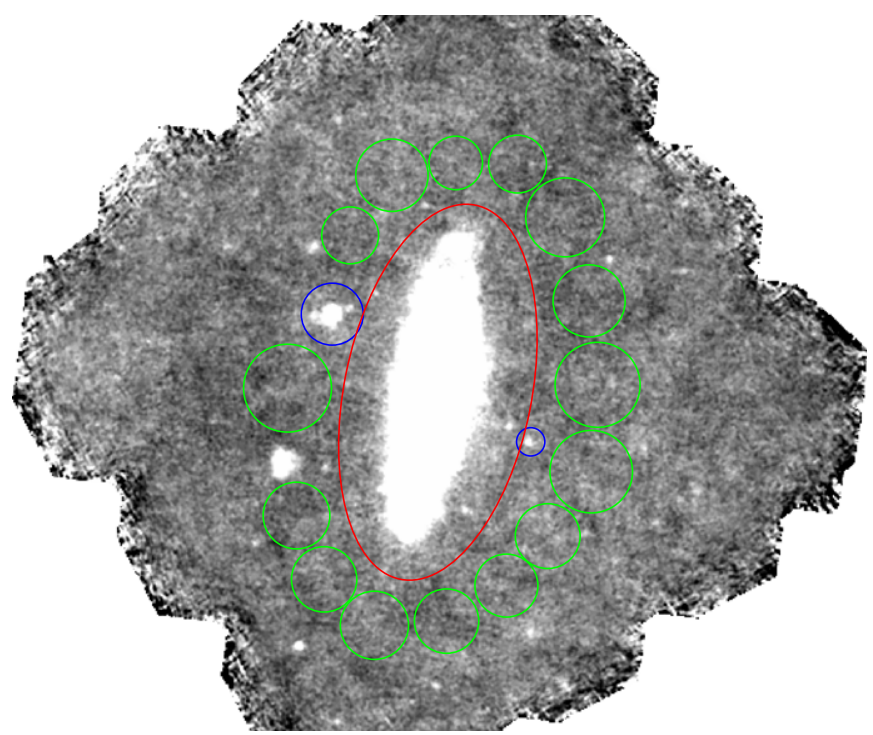

Figure 1. PACS $160 \mu \mathrm{m}$ image of KINGFISH galaxy NGC 7331. The large red ellipse indicates the photometric aperture listed in Table 1, the green circles show the sky apertures, and the two blue circles identify sources to be removed before the photometry is executed. North is up and east is to the left.

signal, and extracts the drifts from the data themselves, taking advantage of the redundancy built in to the scan observations. With the nominal settings used by the KINGFISH survey, the drifts can be determined on timescales greater than or equal to $0.7 \mathrm{~s}$ at 70 and $100 \mu \mathrm{m}$, and $0.9 \mathrm{~s}$ at $160 \mu \mathrm{m}$ (for a sampling interval of $0.1 \mathrm{~s})$. These timescales correspond to lengths between 1.5 and 2.5 times the beam FWHM, from $160 \mu \mathrm{m}$ to $70 \mu \mathrm{m}$. Second-level deglitching was performed, and the option to detect and mask brightness discontinuities was also used. The data are weighted by the inverse square high-frequency noise of each bolometer in each scan.

The ("Level 2") output of Scanamorphos is in the form of a FITS cube for each filter. The four planes are the signal map, the error map, the map of the drifts that have been subtracted, and the weight map. There is currently no propagation of the errors associated with the successive processing steps in the pipeline. In each pixel the error is defined as the unbiased statistical estimate of the error on the mean. The brightness unit is Jy pixel ${ }^{-1}$, and the pixel size is $\sim 1 / 4$ of the beam FWHM, i.e., $1^{\prime \prime} .4$ at $70 \mu \mathrm{m}, 1^{\prime \prime} .7$ at $100 \mu \mathrm{m}$, and $2^{\prime \prime} .85$ at $160 \mu \mathrm{m}$.

\subsection{SPIRE Data}

SPIRE imaging data were taken in Large-Map mode to an extent tailored to each galaxy's size (out to at least $\sim 1.5$ times the optical size). Either two or four scans were obtained for each galaxy based on its Spitzer/MIPS far-infrared surface brightness. The resulting $1 \sigma$ limiting surface brightnesses are approximately $\sigma_{\text {sky }} \sim 0.7,0.4$, and $0.2 \mathrm{MJy} \mathrm{sr}^{-1}$ at 250, 350, and $500 \mu \mathrm{m}$, respectively, for the fainter subset and $\sqrt{2}$ larger values for the brighter galaxies. Calibration uncertainties for SPIRE data are estimated at $\epsilon_{\text {cal }} / f_{v} \approx 7 \%$, following Version 2.4 (2011 June 7) of the SPIRE Observer's Manual. However, the uncertainties are strongly correlated between the three bands and thus the uncertainty on some SPIRE quantities such as the $f_{v}(250 \mu \mathrm{m}) / f_{v}(500 \mu \mathrm{m})$ flux density ratio, for example, are less than the simplistic $\sqrt{2} \times 7 \%$ expectation.

SPIRE observations for six of our galaxies were obtained in the Herschel Reference Survey (Boselli et al. 2010): NGC 4254,
NGC 4321, NGC 4536, NGC 4569, NGC 4579, and NGC 4725; those observations were not duplicated for KINGFISH. The only notable difference between the SPIRE observations for the Herschel Reference Survey and those for KINGFISH is that three scans were employed (versus either two or four for KINGFISH observations, as described above).

The raw SPIRE data are processed through the early stages of HIPE (Version 5.0) to fit slopes to the data ramps and to calibrate the data in physical units. A line is fit to the data for each scan leg after masking out the galaxy, and this fit is subtracted from the data. Discrepant data (usually due to a rogue bolometer, of which there are $<1$ per map) are also masked, and the data are mosaicked using the mapper in HIPE. The map coordinates are then adjusted so that the position of the point sources (measured using StarFinder; Diolaiti et al. 2000) matches those in the MIPS $24 \mu \mathrm{m}$ images. Finally, the images are converted to surface brightness units by dividing by the beam areas published in the SPIRE Observer's Manual: 423, 751, and $1587 \square^{\prime \prime}$ at 250, 350 , and $500 \mu \mathrm{m}$, respectively. Pixel sizes are $6^{\prime \prime}, 10^{\prime \prime}$, and $14^{\prime \prime}$ at 250,350 , and $500 \mu \mathrm{m}$, respectively.

\subsection{Sky Subtraction}

At far-infrared/submillimeter wavelengths the emission from the sky (above the atmosphere) largely comes from Milky Way cirrus and background galaxies. However, the bolometer arrays of Herschel are not absolute photometers, and thus any map produced by any software is the superposition of an estimate of the true sky emission and an unknown (large) offset. Hereafter this superposition is referred to as simply the "sky." While the post-pipeline-processed KINGFISH SPIRE and PACS images have their overall sky levels removed to zeroth order, a procedure has been adopted to remove a more refined local sky value for each galaxy. To accomplish this local sky subtraction, for each PACS and SPIRE image a set of sky apertures has been defined that collectively circumscribes the galaxy, projected on the sky close enough to the galaxy to measure the "local" sky but far enough away to avoid containing any galaxy emission (Figure 1). The emission from any prominent neighboring and/or background galaxies that are projected to lie within the sky apertures is removed before the sky is estimated. The total sky area, derived from the sum of the areas from all sky apertures, is typically significantly greater than that covered by the galaxy aperture itself, thereby limiting the contribution of uncertainty in the sky level to the overall error budget. The mean sky level per pixel is computed from the collection of these sky apertures, the value is scaled to the number of pixels in the galaxy aperture, and the result is subtracted off from the overall galaxy aperture counts (all done within IRAF/IMCNTS).

G. Aniano et al. (2012, in preparation) follow a different procedure for subtracting the sky emission from KINGFISH imaging, including fitting a tilted plane to the sky for each galaxy instead of a single value approach adopted here. G. Aniano et al. (2012, in preparation) study the spatial variations in the farinfrared/submillimeter spectral energy distributions and thus a more detailed characterization of the local sky is necessary. The effects of most sky gradients cancel out in extracting spatially integrated fluxes; the two approaches yield generally consistent global fluxes.

\subsection{Aperture Photometry}

The elliptical apertures used for global photometry are listed in Table 1. The apertures are chosen by eye to encompass 
Table 1

Galaxy Sample

\begin{tabular}{|c|c|c|c|c|c|c|c|c|c|}
\hline Galaxy & $\begin{array}{l}\text { Alternative } \\
\text { Name }\end{array}$ & $\begin{array}{c}\text { Optical } \\
\text { Morphology }\end{array}$ & $\begin{array}{c}E(B-V) \\
\quad(\mathrm{mag})\end{array}$ & $\begin{array}{c}\alpha_{0} \text { and } \delta_{0} \\
(\mathrm{~J} 2000)\end{array}$ & $\begin{array}{c}D_{25} \\
\left({ }^{\prime}\right)\end{array}$ & $\begin{array}{l}2 a \\
\left({ }^{\prime \prime}\right)\end{array}$ & $\begin{array}{l}2 b \\
\left({ }^{\prime \prime}\right)\end{array}$ & $\begin{array}{c}\text { P.A. } \\
\left({ }^{\circ}\right)\end{array}$ & $\begin{array}{c}\log (\mathrm{TIR}) \\
\left(L_{\odot}\right)\end{array}$ \\
\hline NGC0337 & & SBd & 0.112 & $005950.7-073444$ & 2.9 & 253 & 194 & 140 & 10.1 \\
\hline NGC0584 & & $\mathrm{E} 4$ & 0.042 & $013120.6-065205$ & 4.2 & 326 & 278 & 60 & 8.8 \\
\hline NGC0628 & UGC01149 & SAc & 0.070 & $013642.4+154711$ & 10.5 & 879 & 808 & 90 & 9.9 \\
\hline NGC0855 & UGC01718 & E & 0.071 & $021403.7+275237$ & 2.6 & 259 & 169 & 60 & 8.6 \\
\hline NGC0925 & UGC01913 & SABd & 0.076 & $022713.6+333504$ & 10.5 & 735 & 486 & 105 & 9.7 \\
\hline NGC1097 & UGCA041 & $\mathrm{SBb}$ & 0.027 & $024618.0-301642$ & 9.3 & 758 & 612 & 130 & 10.7 \\
\hline NGC1266 & & SB0 & 0.098 & $031600.7-022541$ & 1.5 & 234 & 232 & 0 & 10.4 \\
\hline NGC1291 & & $\mathrm{SB} 0 / \mathrm{a}$ & 0.013 & $031717.9-410616$ & 9.8 & 884 & 836 & 90 & 9.5 \\
\hline NGC1316 & FornaxA & SAB0 & 0.021 & $032241.2-371210$ & 12.0 & 864 & 583 & 50 & 9.9 \\
\hline NGC1377 & & So & 0.028 & $033639.0-205408$ & 1.8 & 181 & 162 & 90 & 10.1 \\
\hline NGC1404 & & E1 & 0.011 & $033852.3-353540$ & 3.3 & 524 & 369 & 149 & $\ldots$ \\
\hline IC0342 & UGC02847 & SABcd & 0.558 & $034648.5+680538$ & 21.4 & 1621 & 1433 & 100 & 10.1 \\
\hline NGC1482 & & SA0 & 0.040 & $035439.0-203009$ & 2.5 & 349 & 310 & 119 & 10.6 \\
\hline NGC1512 & & $\mathrm{SBab}$ & 0.011 & $040355.6-432149$ & 8.9 & 1001 & 928 & 83 & 9.5 \\
\hline NGC2146 & UGC03429 & Sbab & 0.096 & $061835.6+782129$ & 6.0 & 236 & 235 & 120 & 11.0 \\
\hline HoII & UGC04305 & $\operatorname{Im}$ & 0.032 & $081910.8+704320$ & 7.9 & 554 & 465 & 60 & 7.8 \\
\hline DDO053 & UGC04459 & $\operatorname{Im}$ & 0.038 & $083407.4+661043$ & 1.5 & 155 & 142 & 90 & 7.0 \\
\hline NGC2798 & UGC04905 & $\mathrm{SBa}$ & 0.020 & $091723.1+415957$ & 2.6 & 235 & 232 & 90 & 10.6 \\
\hline NGC2841 & UGC04966 & $\mathrm{SAb}$ & 0.015 & $092203.3+505837$ & 8.1 & 629 & 334 & 150 & 10.1 \\
\hline NGC2915 & & I0 & 0.275 & $092609.4-763736$ & 1.9 & 183 & 132 & 110 & 7.6 \\
\hline HoI & UGC05139 & $\mathrm{IABm}$ & 0.050 & $094033.6+711120$ & 3.6 & 264 & 219 & 63 & 7.1 \\
\hline NGC2976 & UGC05221 & SAc & 0.071 & $094715.3+675509$ & 5.9 & 541 & 353 & 144 & 8.9 \\
\hline NGC3049 & UGC05325 & $\mathrm{SBab}$ & 0.038 & $095449.6+091614$ & 2.2 & 218 & 160 & 29 & 9.5 \\
\hline NGC3077 & UGC05398 & I0pec & 0.067 & $100317.5+684354$ & 5.4 & 488 & 436 & 64 & 8.9 \\
\hline M81dwB & UGC05423 & Im & 0.081 & $100531.2+702151$ & 0.9 & 134 & 90 & 139 & 6.5 \\
\hline NGC3190 & UGC05559 & SAap & 0.025 & $101805.7+214957$ & 4.4 & 334 & 196 & 117 & 9.9 \\
\hline NGC3184 & UGC05557 & SABcd & 0.017 & $101815.6+412542$ & 7.4 & 614 & 538 & 169 & 10.0 \\
\hline NGC3198 & UGC05572 & $\mathrm{SBc}$ & 0.012 & $101954.8+453301$ & 8.5 & 518 & 315 & 35 & 10.0 \\
\hline IC2574 & UGC05666 & $\mathrm{SABm}$ & 0.036 & $102823.9+682505$ & 13.2 & 864 & 486 & 59 & 8.3 \\
\hline NGC3265 & UGC05705 & E & 0.024 & $103106.8+284751$ & 1.3 & 184 & 175 & 50 & 9.4 \\
\hline NGC3351 & M095 & $\mathrm{SBb}$ & 0.028 & $104358.1+114210$ & 7.4 & 592 & 441 & 11 & 9.9 \\
\hline NGC3521 & UGC06150 & SABbc & 0.057 & $110548.1-000127$ & 11.0 & 926 & 455 & 165 & 10.5 \\
\hline NGC3621 & UGCA232 & SAd & 0.081 & $111818.3-324855$ & 12.3 & 791 & 555 & 160 & 9.9 \\
\hline NGC3627 & M066 & $\mathrm{SABb}$ & 0.033 & $112013.4+125927$ & 9.1 & 745 & 486 & 167 & 10.4 \\
\hline NGC3773 & UGC06605 & SA0 & 0.027 & $113813.1+120644$ & 1.2 & 118 & 116 & 0 & 8.8 \\
\hline NGC3938 & UGC06856 & SAc & 0.021 & $115250.3+440715$ & 5.4 & 504 & 468 & 0 & 10.3 \\
\hline NGC4236 & UGC07306 & SBdm & 0.015 & $121643.2+692719$ & 21.9 & 1240 & 369 & 162 & 8.7 \\
\hline NGC4254 & M099 & $\mathrm{SAc}$ & 0.039 & $121849.7+142519$ & 5.4 & 519 & 420 & 60 & 10.6 \\
\hline NGC4321 & M100 & SABbc & 0.026 & $122254.8+154907$ & 7.4 & 558 & 483 & 40 & 10.5 \\
\hline NGC4536 & UGC07732 & SABbc & 0.018 & $123427.5+021113$ & 7.6 & 454 & 376 & 120 & 10.3 \\
\hline NGC4559 & UGC07766 & SABcd & 0.018 & $123558.1+275752$ & 10.7 & 576 & 327 & 140 & 9.5 \\
\hline NGC4569 & M090 & SABab & 0.047 & $123650.2+131001$ & 9.5 & 593 & 327 & 21 & 9.7 \\
\hline NGC4579 & M058 & $\mathrm{SABb}$ & 0.041 & $123743.8+114858$ & 5.9 & 325 & 271 & 90 & 10.1 \\
\hline NGC4594 & M104 & SAa & 0.051 & $123959.6-113726$ & 8.7 & 767 & 669 & 90 & 9.6 \\
\hline NGC4625 & UGC07861 & SABmp & 0.018 & $124154.8+411623$ & 2.2 & 298 & 214 & 100 & 8.8 \\
\hline NGC4631 & UGC07865 & SBd & 0.017 & $124204.2+323219$ & 15.5 & 901 & 240 & 85 & 10.4 \\
\hline NGC4725 & UGC07989 & SABab & 0.012 & $125027.7+252948$ & 10.7 & 689 & 523 & 30 & 9.9 \\
\hline NGC4736 & M094 & SAab & 0.018 & $125055.2+410652$ & 11.2 & 944 & 899 & 0 & 9.8 \\
\hline DDO154 & UGC08024 & $\mathrm{IBm}$ & 0.009 & $125407.6+270916$ & 3.0 & 216 & 126 & 50 & $\ldots$ \\
\hline NGC4826 & M064 & SAab & 0.041 & $125643.3+214048$ & 10.0 & 716 & 427 & 114 & 9.6 \\
\hline DDO165 & UGC08201 & $\operatorname{Im}$ & 0.024 & $130625.9+674229$ & 3.5 & 263 & 161 & 90 & $\ldots$ \\
\hline NGC5055 & M063 & SAbc & 0.018 & $131549.2+420147$ & 12.6 & 1097 & 711 & 80 & 10.3 \\
\hline NGC5398 & Tololo89 & SBdm & 0.066 & $140121.2-330402$ & 2.8 & 198 & 146 & 0 & 8.6 \\
\hline NGC5457 & M101 & SABcd & 0.009 & $140325.0+542429$ & 28.8 & 1800 & 1446 & 37 & 10.4 \\
\hline NGC5408 & & $\mathrm{IBm}$ & 0.068 & $140321.1-412241$ & 1.6 & 256 & 209 & 67 & 8.3 \\
\hline NGC5474 & UGC09013 & SAcd & 0.011 & $140500.8+533920$ & 4.8 & 412 & 373 & 90 & 8.7 \\
\hline NGC5713 & UGC09451 & SABbcp & 0.039 & $144011.4-001726$ & 2.8 & 225 & 225 & 90 & 10.5 \\
\hline NGC5866 & UGC09723 & So & 0.013 & $150628.8+554551$ & 4.7 & 500 & 306 & 129 & 9.8 \\
\hline NGC6946 & UGC11597 & SABcd & 0.342 & $203449.2+600959$ & 11.5 & 953 & 928 & 0 & 10.5 \\
\hline NGC7331 & UGC12113 & $\mathrm{SAb}$ & 0.091 & $223704.3+342435$ & 10.5 & 683 & 335 & 168 & 10.7 \\
\hline NGC7793 & & SAd & 0.019 & $235749.9-323525$ & 9.3 & 716 & 526 & 98 & 9.3 \\
\hline
\end{tabular}

Notes. $D_{25}$ is the diameter of the $B$ band isophote defined at $25 \mathrm{mag} \operatorname{arcsec}^{-2} .2 a$ and $2 b$ are the lengths of the major and minor axes used in the elliptical aperture photometry described herein; the position angle of the aperture's major axis is measured east of north. The total infrared listed in the last column is derived from Equation (4) of Dale \& Helou (2002) and the far-infrared fluxes in Dale et al. (2007, 2009) and from Engelbracht et al. (2008) for IC 0342 and NGC 2146. 
Table 2

Far-infrared/Submillimeter Flux Densities

\begin{tabular}{|c|c|c|c|c|c|c|}
\hline Galaxy & $\begin{array}{c}\text { PACS } \\
70 \mu \mathrm{m} \\
(\mathrm{Jy})\end{array}$ & $\begin{array}{c}\text { PACS } \\
100 \mu \mathrm{m} \\
(\mathrm{Jy})\end{array}$ & $\begin{array}{c}\text { PACS } \\
160 \mu \mathrm{m} \\
(\mathrm{Jy})\end{array}$ & $\begin{array}{l}\text { SPIRE } \\
250 \mu \mathrm{m} \\
(\mathrm{Jy})\end{array}$ & $\begin{array}{c}\text { SPIRE } \\
350 \mathrm{~m} \\
(\mathrm{Jy})\end{array}$ & $\begin{array}{l}\text { SPIRE } \\
500 \mu \mathrm{m} \\
(\mathrm{Jy})\end{array}$ \\
\hline NGC0337 & $1.30 \pm 0.07 \mathrm{E}+1$ & $1.95 \pm 0.10 \mathrm{E}+1$ & $1.96 \pm 0.10 \mathrm{E}+1$ & $9.79 \pm 0.70 \mathrm{E}+0$ & $4.37 \pm 0.31 \mathrm{E}+0$ & $1.88 \pm 0.14 \mathrm{E}+0$ \\
\hline NGC0584 & & & & $<8.84 \mathrm{E}-1$ & $<8.19 \mathrm{E}-1$ & $<7.63 \mathrm{E}-1$ \\
\hline NGC0628 & $3.67 \pm 0.18 \mathrm{E}+1$ & $7.40 \pm 0.37 \mathrm{E}+1$ & $1.16 \pm 0.06 \mathrm{E}+2$ & $6.55 \pm 0.47 \mathrm{E}+1$ & $3.06 \pm 0.22 \mathrm{E}+1$ & $1.33 \pm 0.10 \mathrm{E}+1$ \\
\hline NGC0855 & $2.30 \pm 0.12 \mathrm{E}+0$ & $2.04 \pm 0.12 \mathrm{E}+0$ & $2.16 \pm 0.12 \mathrm{E}+0$ & $1.48 \pm 0.11 \mathrm{E}+0$ & $7.47 \pm 0.65 \mathrm{E}-1$ & $2.66 \pm 0.40 \mathrm{E}-1$ \\
\hline NGC0925 & $1.08 \pm 0.06 \mathrm{E}+1$ & $2.47 \pm 0.12 \mathrm{E}+1$ & $3.65 \pm 0.18 \mathrm{E}+1$ & $2.77 \pm 0.20 \mathrm{E}+1$ & $1.48 \pm 0.11 \mathrm{E}+1$ & $8.03 \pm 0.58 \mathrm{E}+0$ \\
\hline NGC1097 & $7.75 \pm 0.39 \mathrm{E}+1$ & $1.16 \pm 0.06 \mathrm{E}+2$ & $1.34 \pm 0.07 \mathrm{E}+2$ & $7.22 \pm 0.51 \mathrm{E}+1$ & $3.08 \pm 0.22 \mathrm{E}+1$ & $1.26 \pm 0.09 \mathrm{E}+1$ \\
\hline NGC1266 & $1.45 \pm 0.07 \mathrm{E}+1$ & $1.59 \pm 0.08 \mathrm{E}+1$ & $1.13 \pm 0.06 \mathrm{E}+1$ & $4.38 \pm 0.31 \mathrm{E}+0$ & $1.60 \pm 0.12 \mathrm{E}+0$ & $5.32 \pm 0.55 \mathrm{E}-1$ \\
\hline NGC1291 & $5.26 \pm 0.32 \mathrm{E}+0$ & $1.28 \pm 0.07 \mathrm{E}+1$ & $2.03 \pm 0.11 \mathrm{E}+1$ & $1.59 \pm 0.11 \mathrm{E}+1$ & $7.98 \pm 0.59 \mathrm{E}+0$ & $3.52 \pm 0.29 \mathrm{E}+0$ \\
\hline NGC1316 & $5.81 \pm 0.33 \mathrm{E}+0$ & $9.30 \pm 0.50 \mathrm{E}+0$ & $1.15 \pm 0.06 \mathrm{E}+1$ & $4.80 \pm 0.37 \mathrm{E}+0$ & $2.06 \pm 0.19 \mathrm{E}+0$ & $8.16 \pm 1.33 \mathrm{E}-1$ \\
\hline NGC1377 & $6.89 \pm 0.35 \mathrm{E}+0$ & $5.85 \pm 0.30 \mathrm{E}+0$ & $3.38 \pm 0.19 \mathrm{E}+0$ & $1.32 \pm 0.10 \mathrm{E}+0$ & $4.94 \pm 0.47 \mathrm{E}-1$ & $1.99 \pm 0.32 \mathrm{E}-1$ \\
\hline NGC1404 & $<6.71 \mathrm{E}-1$ & $<7.06 \mathrm{E}-1$ & $<1.02 \mathrm{E} 0$ & $<4.30 \mathrm{E}-1$ & $<3.99 \mathrm{E}-1$ & $<3.72 \mathrm{E}-1$ \\
\hline IC0342 & $4.48 \pm 0.22 \mathrm{E}+2$ & $8.47 \pm 0.42 \mathrm{E}+2$ & $1.11 \pm 0.06 \mathrm{E}+3$ & $5.95 \pm 0.42 \mathrm{E}+2$ & $2.61 \pm 0.19 \mathrm{E}+2$ & $1.02 \pm 0.07 \mathrm{E}+2$ \\
\hline NGC1482 & $4.07 \pm 0.20 \mathrm{E}+1$ & $4.95 \pm 0.25 \mathrm{E}+1$ & $4.20 \pm 0.21 \mathrm{E}+1$ & $1.68 \pm 0.12 \mathrm{E}+1$ & $6.35 \pm 0.45 \mathrm{E}+0$ & $2.21 \pm 0.17 \mathrm{E}+0$ \\
\hline NGC1512 & $7.99 \pm 0.47 \mathrm{E}+0$ & $1.38 \pm 0.07 \mathrm{E}+1$ & $1.87 \pm 0.10 \mathrm{E}+1$ & $1.56 \pm 0.11 \mathrm{E}+1$ & $8.66 \pm 0.64 \mathrm{E}+0$ & $4.20 \pm 0.34 \mathrm{E}+0$ \\
\hline NGC2146 & $1.98 \pm 0.10 \mathrm{E}+2$ & $2.32 \pm 0.12 \mathrm{E}+2$ & $1.81 \pm 0.09 \mathrm{E}+2$ & $6.55 \pm 0.47 \mathrm{E}+1$ & $2.33 \pm 0.17 \mathrm{E}+1$ & $7.45 \pm 0.53 \mathrm{E}+0$ \\
\hline HoII & $3.18 \pm 0.35 \mathrm{E}+0$ & $3.89 \pm 0.45 \mathrm{E}+0$ & $3.86 \pm 0.63 \mathrm{E}+0$ & $1.82 \pm 0.16 \mathrm{E}+0$ & $8.04 \pm 1.08 \mathrm{E}-1$ & $3.37 \pm 1.69 \mathrm{E}-1$ \\
\hline DDO053 & $3.90 \pm 0.42 \mathrm{E}-1$ & $4.80 \pm 1.21 \mathrm{E}-1$ & $2.50 \pm 1.77 \mathrm{E}-1$ & $1.86 \pm 0.32 \mathrm{E}-1$ & $9.99 \pm 2.81 \mathrm{E}-2$ & $<1.25 \mathrm{E}-1$ \\
\hline NGC2798 & $2.42 \pm 0.12 \mathrm{E}+1$ & $2.73 \pm 0.14 \mathrm{E}+1$ & $2.06 \pm 0.10 \mathrm{E}+1$ & $8.02 \pm 0.57 \mathrm{E}+0$ & $2.90 \pm 0.21 \mathrm{E}+0$ & $1.08 \pm 0.09 \mathrm{E}+0$ \\
\hline NGC2841 & $9.49 \pm 0.49 \mathrm{E}+0$ & $2.57 \pm 0.13 \mathrm{E}+1$ & $4.95 \pm 0.25 \mathrm{E}+1$ & $3.49 \pm 0.25 \mathrm{E}+1$ & $1.60 \pm 0.11 \mathrm{E}+1$ & $7.01 \pm 0.50 \mathrm{E}+0$ \\
\hline NGC2915 & $1.01 \pm 0.06 \mathrm{E}+0$ & $1.66 \pm 0.09 \mathrm{E}+0$ & $1.46 \pm 0.11 \mathrm{E}+0$ & $9.28 \pm 0.73 \mathrm{E}-1$ & $5.28 \pm 0.47 \mathrm{E}-1$ & $2.54 \pm 0.32 \mathrm{E}-1$ \\
\hline HoI & $3.71 \pm 0.62 \mathrm{E}-1$ & $4.21 \pm 0.70 \mathrm{E}-1$ & $3.72 \pm 1.20 \mathrm{E}-1$ & $3.56 \pm 0.53 \mathrm{E}-1$ & $2.23 \pm 0.47 \mathrm{E}-1$ & $1.35 \pm 0.41 \mathrm{E}-1$ \\
\hline NGC2976 & $1.92 \pm 0.10 \mathrm{E}+1$ & $3.58 \pm 0.18 \mathrm{E}+1$ & $4.64 \pm 0.23 \mathrm{E}+1$ & $2.50 \pm 0.18 \mathrm{E}+1$ & $1.17 \pm 0.08 \mathrm{E}+1$ & $4.79 \pm 0.35 \mathrm{E}+0$ \\
\hline NGC3049 & $3.40 \pm 0.18 \mathrm{E}+0$ & $4.59 \pm 0.23 \mathrm{E}+0$ & $4.54 \pm 0.24 \mathrm{E}+0$ & $2.80 \pm 0.20 \mathrm{E}+0$ & $1.41 \pm 0.11 \mathrm{E}+0$ & $7.97 \pm 0.65 \mathrm{E}-1$ \\
\hline NGC3077 & $2.04 \pm 0.10 \mathrm{E}+1$ & $2.79 \pm 0.14 \mathrm{E}+1$ & $2.83 \pm 0.14 \mathrm{E}+1$ & $1.43 \pm 0.10 \mathrm{E}+1$ & $6.47 \pm 0.47 \mathrm{E}+0$ & $2.89 \pm 0.22 \mathrm{E}+0$ \\
\hline M81dwB & $1.21 \pm 0.41 \mathrm{E}-1$ & $2.01 \pm 0.31 \mathrm{E}-1$ & $2.42 \pm 0.82 \mathrm{E}-1$ & $1.87 \pm 0.25 \mathrm{E}-1$ & $1.03 \pm 0.22 \mathrm{E}-1$ & $5.66 \pm 2.83 \mathrm{E}-2$ \\
\hline NGC3190 & $6.30 \pm 0.33 \mathrm{E}+0$ & $1.06 \pm 0.05 \mathrm{E}+1$ & $1.54 \pm 0.08 \mathrm{E}+1$ & $8.88 \pm 0.63 \mathrm{E}+0$ & $3.71 \pm 0.27 \mathrm{E}+0$ & $1.38 \pm 0.11 \mathrm{E}+0$ \\
\hline NGC3184 & $1.55 \pm 0.08 \mathrm{E}+1$ & $3.47 \pm 0.17 \mathrm{E}+1$ & $5.49 \pm 0.28 \mathrm{E}+1$ & $3.43 \pm 0.24 \mathrm{E}+1$ & $1.53 \pm 0.11 \mathrm{E}+1$ & $6.73 \pm 0.49 \mathrm{E}+0$ \\
\hline NGC3198 & $9.75 \pm 0.51 \mathrm{E}+0$ & $2.00 \pm 0.10 \mathrm{E}+1$ & $2.99 \pm 0.15 \mathrm{E}+1$ & $1.96 \pm 0.14 \mathrm{E}+1$ & $9.95 \pm 0.71 \mathrm{E}+0$ & $4.74 \pm 0.34 \mathrm{E}+0$ \\
\hline IC2574 & $5.61 \pm 0.37 \mathrm{E}+0$ & $7.57 \pm 0.42 \mathrm{E}+0$ & $9.61 \pm 0.53 \mathrm{E}+0$ & $7.16 \pm 0.52 \mathrm{E}+0$ & $4.83 \pm 0.36 \mathrm{E}+0$ & $2.13 \pm 0.19 \mathrm{E}+0$ \\
\hline NGC3265 & $2.47 \pm 0.13 \mathrm{E}+0$ & $3.10 \pm 0.16 \mathrm{E}+0$ & $2.63 \pm 0.15 \mathrm{E}+0$ & $1.24 \pm 0.10 \mathrm{E}+0$ & $5.51 \pm 0.51 \mathrm{E}-1$ & $2.38 \pm 0.35 \mathrm{E}-1$ \\
\hline NGC3351 & $2.53 \pm 0.13 \mathrm{E}+1$ & $4.61 \pm 0.23 \mathrm{E}+1$ & $5.51 \pm 0.28 \mathrm{E}+1$ & $3.24 \pm 0.23 \mathrm{E}+1$ & $1.37 \pm 0.10 \mathrm{E}+1$ & $5.32 \pm 0.39 \mathrm{E}+0$ \\
\hline NGC3521 & $7.85 \pm 0.39 \mathrm{E}+1$ & $1.58 \pm 0.08 \mathrm{E}+2$ & $2.10 \pm 0.10 \mathrm{E}+2$ & $1.14 \pm 0.08 \mathrm{E}+2$ & $4.72 \pm 0.34 \mathrm{E}+1$ & $1.94 \pm 0.14 \mathrm{E}+1$ \\
\hline NGC3621 & $4.95 \pm 0.25 \mathrm{E}+1$ & $9.44 \pm 0.47 \mathrm{E}+1$ & $1.28 \pm 0.06 \mathrm{E}+2$ & $7.12 \pm 0.51 \mathrm{E}+1$ & $3.17 \pm 0.23 \mathrm{E}+1$ & $1.46 \pm 0.10 \mathrm{E}+1$ \\
\hline NGC3627 & $1.04 \pm 0.05 \mathrm{E}+2$ & $1.79 \pm 0.09 \mathrm{E}+2$ & $2.02 \pm 0.10 \mathrm{E}+2$ & $9.67 \pm 0.69 \mathrm{E}+1$ & $3.76 \pm 0.27 \mathrm{E}+1$ & $1.44 \pm 0.10 \mathrm{E}+1$ \\
\hline NGC3773 & $1.29 \pm 0.08 \mathrm{E}+0$ & $1.85 \pm 0.11 \mathrm{E}+0$ & $1.91 \pm 0.14 \mathrm{E}+0$ & $1.06 \pm 0.08 \mathrm{E}+0$ & $4.34 \pm 0.38 \mathrm{E}-1$ & $1.80 \pm 0.24 \mathrm{E}-1$ \\
\hline NGC3938 & $1.58 \pm 0.08 \mathrm{E}+1$ & $2.86 \pm 0.15 \mathrm{E}+1$ & $3.96 \pm 0.20 \mathrm{E}+1$ & $2.37 \pm 0.17 \mathrm{E}+1$ & $1.03 \pm 0.07 \mathrm{E}+1$ & $4.34 \pm 0.32 \mathrm{E}+0$ \\
\hline NGC4236 & $7.46 \pm 0.46 \mathrm{E}+0$ & $1.23 \pm 0.07 \mathrm{E}+1$ & $1.85 \pm 0.11 \mathrm{E}+1$ & $1.16 \pm 0.08 \mathrm{E}+1$ & $7.37 \pm 0.54 \mathrm{E}+0$ & $4.21 \pm 0.32 \mathrm{E}+0$ \\
\hline NGC4254 ${ }^{\mathrm{a}}$ & $5.64 \pm 0.28 \mathrm{E}+1$ & $1.06 \pm 0.05 \mathrm{E}+2$ & $1.30 \pm 0.07 \mathrm{E}+2$ & $6.57 \pm 0.47 \mathrm{E}+1$ & $2.66 \pm 0.19 \mathrm{E}+1$ & $9.16 \pm 0.66 \mathrm{E}+0$ \\
\hline NGC4321 ${ }^{\mathrm{a}}$ & $4.12 \pm 0.21 \mathrm{E}+1$ & $8.55 \pm 0.43 \mathrm{E}+1$ & $1.20 \pm 0.06 \mathrm{E}+2$ & $6.76 \pm 0.48 \mathrm{E}+1$ & $2.79 \pm 0.20 \mathrm{E}+1$ & $1.08 \pm 0.08 \mathrm{E}+1$ \\
\hline NGC4536 ${ }^{\mathrm{a}}$ & $3.89 \pm 0.20 \mathrm{E}+1$ & $5.26 \pm 0.26 \mathrm{E}+1$ & $5.55 \pm 0.28 \mathrm{E}+1$ & $2.88 \pm 0.20 \mathrm{E}+1$ & $1.26 \pm 0.09 \mathrm{E}+1$ & $5.53 \pm 0.40 \mathrm{E}+0$ \\
\hline NGC4559 & $1.59 \pm 0.08 \mathrm{E}+1$ & $3.10 \pm 0.16 \mathrm{E}+1$ & $4.10 \pm 0.21 \mathrm{E}+1$ & $2.55 \pm 0.18 \mathrm{E}+1$ & $1.28 \pm 0.09 \mathrm{E}+1$ & $6.37 \pm 0.46 \mathrm{E}+0$ \\
\hline NGC4569a & $1.46 \pm 0.07 \mathrm{E}+1$ & $3.04 \pm 0.15 \mathrm{E}+1$ & $4.03 \pm 0.20 \mathrm{E}+1$ & $2.24 \pm 0.16 \mathrm{E}+1$ & $9.41 \pm 0.67 \mathrm{E}+0$ & $3.67 \pm 0.27 \mathrm{E}+0$ \\
\hline NGC4579a & $9.94 \pm 0.51 \mathrm{E}+0$ & $2.33 \pm 0.12 \mathrm{E}+1$ & $3.54 \pm 0.18 \mathrm{E}+1$ & $2.12 \pm 0.15 \mathrm{E}+1$ & $8.87 \pm 0.63 \mathrm{E}+0$ & $3.54 \pm 0.26 \mathrm{E}+0$ \\
\hline NGC4594 & $7.87 \pm 0.49 \mathrm{E}+0$ & $2.39 \pm 0.13 \mathrm{E}+1$ & $3.89 \pm 0.20 \mathrm{E}+1$ & $2.56 \pm 0.18 \mathrm{E}+1$ & $1.21 \pm 0.09 \mathrm{E}+1$ & $5.56 \pm 0.41 \mathrm{E}+0$ \\
\hline NGC4625 & $1.36 \pm 0.12 \mathrm{E}+0$ & $3.04 \pm 0.20 \mathrm{E}+0$ & $4.48 \pm 0.23 \mathrm{E}+0$ & $2.81 \pm 0.21 \mathrm{E}+0$ & $1.40 \pm 0.11 \mathrm{E}+0$ & $6.44 \pm 0.62 \mathrm{E}-1$ \\
\hline NGC4631 & $1.37 \pm 0.07 \mathrm{E}+2$ & $2.23 \pm 0.11 \mathrm{E}+2$ & $2.46 \pm 0.12 \mathrm{E}+2$ & $1.24 \pm 0.09 \mathrm{E}+2$ & $5.45 \pm 0.39 \mathrm{E}+1$ & $2.40 \pm 0.17 \mathrm{E}+1$ \\
\hline NGC4725 ${ }^{\mathrm{a}}$ & $7.93 \pm 0.46 \mathrm{E}+0$ & $2.28 \pm 0.12 \mathrm{E}+1$ & $4.66 \pm 0.23 \mathrm{E}+1$ & $3.27 \pm 0.23 \mathrm{E}+1$ & $1.66 \pm 0.12 \mathrm{E}+1$ & $7.93 \pm 0.57 \mathrm{E}+0$ \\
\hline NGC4736 & $1.03 \pm 0.05 \mathrm{E}+2$ & $1.59 \pm 0.08 \mathrm{E}+2$ & $1.45 \pm 0.07 \mathrm{E}+2$ & $7.04 \pm 0.50 \mathrm{E}+1$ & $2.80 \pm 0.20 \mathrm{E}+1$ & $1.18 \pm 0.09 \mathrm{E}+1$ \\
\hline DDO154 & $<3.31 \mathrm{E}-1$ & $<4.27 \mathrm{E}-1$ & $<2.67 \mathrm{E}-1$ & $<1.62 \mathrm{E}-1$ & $<1.50 \mathrm{E}-1$ & $<1.39 \mathrm{E}-1$ \\
\hline NGC4826 & $5.47 \pm 0.27 \mathrm{E}+1$ & $9.57 \pm 0.48 \mathrm{E}+1$ & $9.41 \pm 0.47 \mathrm{E}+1$ & $4.24 \pm 0.30 \mathrm{E}+1$ & $1.64 \pm 0.12 \mathrm{E}+1$ & $6.30 \pm 0.46 \mathrm{E}+0$ \\
\hline DDO165 & $<4.13 \mathrm{E}-1$ & $<5.33 \mathrm{E}-1$ & $<3.33 \mathrm{E}-1$ & $<2.01 \mathrm{E}-1$ & $<1.87 \mathrm{E}-1$ & $<1.74 \mathrm{E}-1$ \\
\hline NGC5055 & $7.34 \pm 0.37 \mathrm{E}+1$ & $1.70 \pm 0.08 \mathrm{E}+2$ & $2.48 \pm 0.12 \mathrm{E}+2$ & $1.50 \pm 0.11 \mathrm{E}+2$ & $6.42 \pm 0.46 \mathrm{E}+1$ & $2.61 \pm 0.19 \mathrm{E}+1$ \\
\hline NGC5398 & $2.19 \pm 0.12 \mathrm{E}+0$ & $2.98 \pm 0.16 \mathrm{E}+0$ & $2.75 \pm 0.16 \mathrm{E}+0$ & $2.03 \pm 0.15 \mathrm{E}+0$ & $1.05 \pm 0.08 \mathrm{E}+0$ & $5.52 \pm 0.49 \mathrm{E}-1$ \\
\hline NGC5457 & $1.23 \pm 0.06 \mathrm{E}+2$ & $2.43 \pm 0.12 \mathrm{E}+2$ & $3.41 \pm 0.17 \mathrm{E}+2$ & $2.08 \pm 0.15 \mathrm{E}+2$ & $9.69 \pm 0.69 \mathrm{E}+1$ & $4.53 \pm 0.32 \mathrm{E}+1$ \\
\hline NGC5408 & $3.60 \pm 0.19 \mathrm{E}+0$ & $2.65 \pm 0.15 \mathrm{E}+0$ & $2.02 \pm 0.11 \mathrm{E}+0$ & $7.85 \pm 0.72 \mathrm{E}-1$ & $3.86 \pm 0.50 \mathrm{E}-1$ & $2.09 \pm 0.42 \mathrm{E}-1$ \\
\hline NGC5474 & $3.24 \pm 0.18 \mathrm{E}+0$ & $4.61 \pm 0.25 \mathrm{E}+0$ & $7.12 \pm 0.37 \mathrm{E}+0$ & $5.37 \pm 0.39 \mathrm{E}+0$ & $2.91 \pm 0.22 \mathrm{E}+0$ & $1.58 \pm 0.13 \mathrm{E}+0$ \\
\hline NGC5713 & $2.89 \pm 0.14 \mathrm{E}+1$ & $4.03 \pm 0.20 \mathrm{E}+1$ & $3.93 \pm 0.20 \mathrm{E}+1$ & $1.68 \pm 0.12 \mathrm{E}+1$ & $6.39 \pm 0.46 \mathrm{E}+0$ & $2.30 \pm 0.17 \mathrm{E}+0$ \\
\hline NGC5866 & $8.12 \pm 0.42 \mathrm{E}+0$ & $1.67 \pm 0.09 \mathrm{E}+1$ & $1.84 \pm 0.10 \mathrm{E}+1$ & $8.04 \pm 0.58 \mathrm{E}+0$ & $3.14 \pm 0.23 \mathrm{E}+0$ & $1.14 \pm 0.10 \mathrm{E}+0$ \\
\hline NGC6946 & $2.46 \pm 0.12 \mathrm{E}+2$ & $4.35 \pm 0.22 \mathrm{E}+2$ & $5.42 \pm 0.27 \mathrm{E}+2$ & $2.74 \pm 0.19 \mathrm{E}+2$ & $1.09 \pm 0.08 \mathrm{E}+2$ & $4.28 \pm 0.30 \mathrm{E}+1$ \\
\hline NGC7331 & $6.53 \pm 0.33 \mathrm{E}+1$ & $1.32 \pm 0.07 \mathrm{E}+2$ & $1.76 \pm 0.09 \mathrm{E}+2$ & $9.53 \pm 0.68 \mathrm{E}+1$ & $4.06 \pm 0.29 \mathrm{E}+1$ & $1.65 \pm 0.12 \mathrm{E}+1$ \\
\hline NGC7793 & $3.20 \pm 0.16 \mathrm{E}+1$ & $6.58 \pm 0.33 \mathrm{E}+1$ & $9.11 \pm 0.46 \mathrm{E}+1$ & $5.63 \pm 0.40 \mathrm{E}+1$ & $2.84 \pm 0.20 \mathrm{E}+1$ & $1.39 \pm 0.10 \mathrm{E}+1$ \\
\hline
\end{tabular}

Notes. The compact table entry format T.UV \pm W.XYEZ implies (T.UV \pm W.XY) $\times 10^{Z}$. See Section 3 for corrections that have been applied to the data. The uncertainties include both statistical and systematic effects. $5 \sigma$ upper limits are provided for non-detections. No color corrections have been applied. PACS observations for NGC0584 are not yet available.

${ }^{\text {a }}$ SPIRE imaging taken from the Herschel Reference Survey (Boselli et al. 2010). 

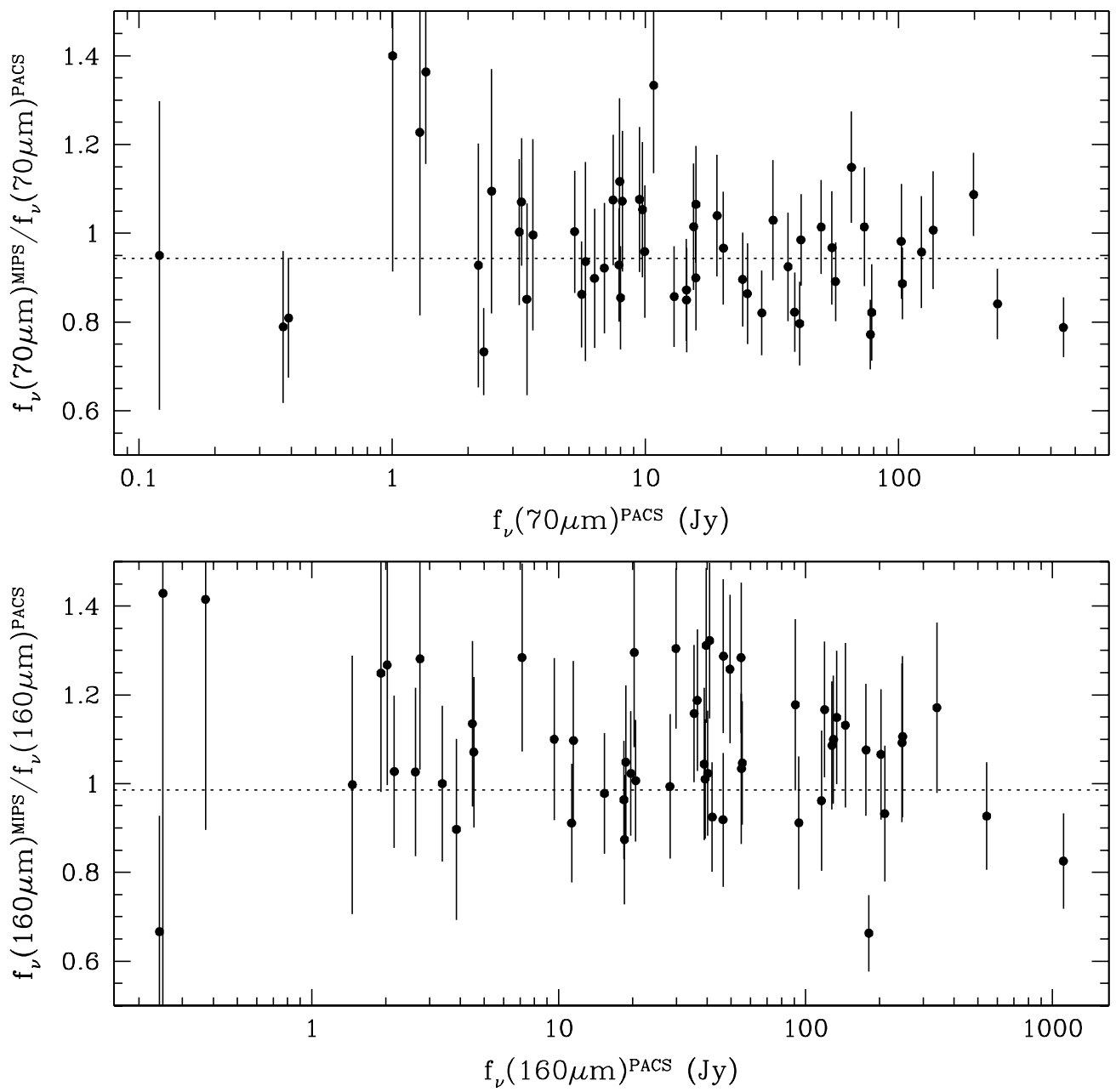

Figure 2. Comparison of spatially integrated Spitzer/MIPS and Herschel/PACS photometry at $70 \mu \mathrm{m}$ (top) and $160 \mu \mathrm{m}$ (bottom) for all sample galaxies. The horizontal dotted lines indicate the ratios corresponding to perfect agreement between data taken by the two observatories after accounting for differences in the Spitzer and Herschel spectral responses and calibration schemes; for typical galaxy spectral energy distributions, the dotted lines differ from unity by a factor of 1.06 and 1.015 for the 70 and $160 \mu \mathrm{m}$ comparisons, respectively.

essentially all of the emission at every wavelength; aperture corrections described below are incorporated to recover the amount of any light that lies beyond these apertures. The average ratio of aperture major axis length $2 a$ to the de Vaucouleurs $D_{25}$ optical major axis is 1.45 (with a $1 \sigma$ dispersion in this ratio of 0.45 ). The same aperture is used to extract the flux at each wavelength studied here, and they are very similar, if not identical, to those used for SINGS photometry (Dale et al. 2007), for the 57 galaxies that overlap the two samples. As a test of the robustness of the aperture choices, the global flux densities using these apertures are compared to the values obtained using apertures with 5\% larger and 5\% smaller semiminor and semimajor axes. The impact of using $\sim 10 \%$ larger or $\sim 10 \%$ smaller aperture areas is a median difference of less than $1 \%$ on the flux densities for all wavelengths.

Prior to extracting fluxes from aperture photometry, any emission from neighboring or background galaxies is identified and removed from the area covered by each aperture. The identification is assisted by ancillary data at shorter wavelengths and higher spatial resolution (e.g., Spitzer/IRAC 3.6 and $8.0 \mu \mathrm{m}$, $H S T$ optical, and ground-based $\mathrm{H} \alpha$ imaging). The removal is accomplished via IRAF/IMEDIT by replacing the values of contaminated pixels with the values from a random selection of nearby sky pixels, thereby incorporating the same noise statistics as the sky. Usually the removal of such emission affects the global flux at less than the $1 \%$ level, but in a few cases the impact is quite important, e.g., NGC 1317 lies within the aperture of NGC 1316, and background galaxies in the fields of the fainter dwarfs like Ho II and DDO 053 would contribute significantly to the integrated flux (by up to $\sim 30 \%-50 \%$ ) if not removed (see also Walter et al. 2007).

Diffraction inevitably results in a small portion of the galaxy emission appearing beyond the chosen apertures, however, and thus aperture corrections are formulated to mitigate this effect. Aperture corrections are empirically determined from a comparison of fluxes from smoothed and unsmoothed Spitzer/IRAC $8.0 \mu \mathrm{m}$ imaging, which has a native resolution of $\sim 1$ 1".8. The aperture correction for a given PACS or SPIRE flux is the ratio of the flux from the unsmoothed $8.0 \mu \mathrm{m}$ image to the flux from the $8.0 \mu \mathrm{m}$ image smoothed to the same point-spread function (PSF) as the Herschel image in question. Due to the typically generous aperture size and sharp Herschel PACS and SPIRE PSFs, the amplitudes of the KINGFISH global photometry aperture corrections are typically quite small, with median values of 1.0 at all wavelengths and maximum values of 1.03 for PACS and between 1.07 and 1.13 for SPIRE. This technique 


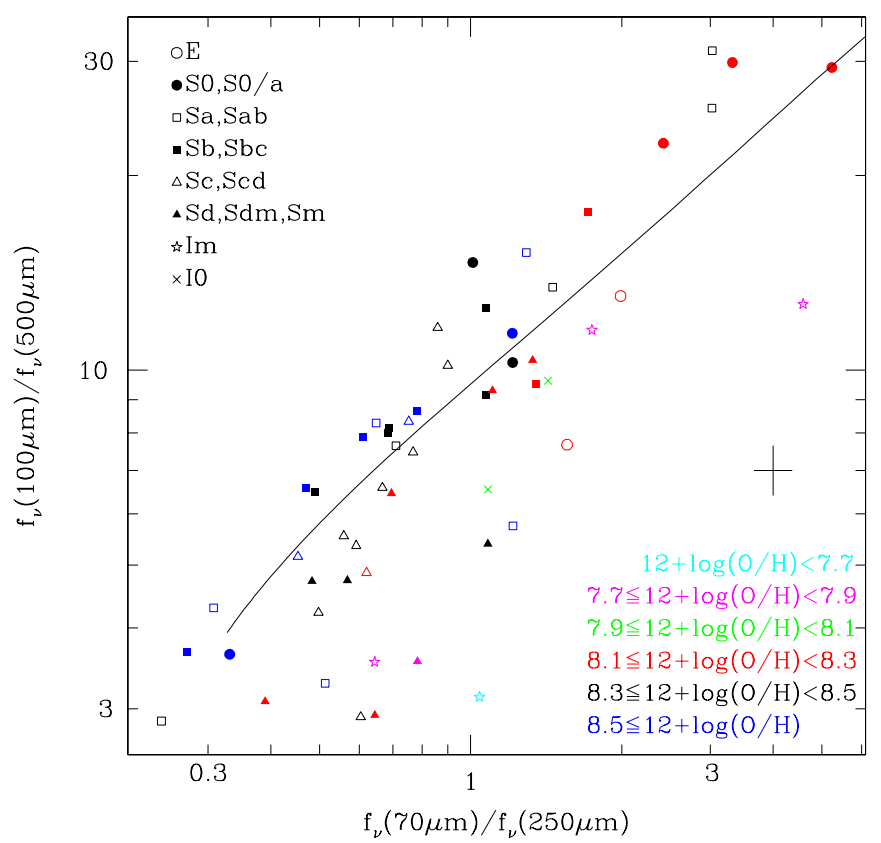

Figure 3. Far-infrared/submillimeter color-color diagram for the KINGFISH sample. "Characteristic" oxygen abundances are taken from Table 9 of Moustakas et al. (2010) using the Pilyugin \& Thuan (2005) metallicity scale; if unavailable in Table 9, then the value is computed using the $B$ luminosity and Equation (10) of Moustakas et al. (2010), which uses the same metallicity scale. The solid line indicates the sequence of model spectral energy distributions of Dale \& Helou (2002) derived from the average global trends for a sample of normal star-forming galaxies observed by ISO and IRAS. A set of typical error bars is provided.

assumes that a galaxy's profile in the far-infrared matches that of its (mostly) PAH profile in the mid-infrared, and there may in fact be appreciable differences in the two emission profiles.

The uncertainties in the integrated photometry $\epsilon_{\text {total }}$ are computed as a combination in quadrature of the calibration uncertainty $\epsilon_{\mathrm{cal}}$ and the measurement uncertainty $\epsilon_{\text {sky }}$ based on the measured sky fluctuations and the areas covered by the galaxy and the sum of the sky apertures, i.e.,

$$
\epsilon_{\mathrm{total}}=\sqrt{\epsilon_{\mathrm{cal}}^{2}+\epsilon_{\mathrm{sky}}^{2}}
$$

with

$$
\epsilon_{\text {sky }}=\sigma_{\text {sky }} \Omega_{\text {pix }} \sqrt{N_{\text {pix }}+N_{\text {pix }}^{2} / N_{\text {sky }}},
$$

where $\sigma_{\text {sky }}$ is the standard deviation of the sky surface brightness fluctuations, $\Omega_{\text {pix }}$ is the solid angle subtended per pixel, and $N_{\text {pix }}$ and $N_{\text {sky }}$ are the number of pixels in the galaxy and (the sum of) the sky apertures, respectively. For the few sources undetected by Herschel imaging, $5 \sigma$ upper limits are derived assuming that a galaxy spans all $N_{\text {pix }}$ pixels in the aperture,

$$
f_{v}(5 \sigma \text { upper limit })=5 \epsilon_{\text {sky }}
$$

\section{RESULTS}

\subsection{Flux Densities}

Table 2 presents the spatially integrated flux densities for all 61 KINGFISH galaxies for all six Herschel photometric bands. The tabulated flux densities include aperture corrections (Section 3.4) and have Galactic extinction (Schlegel et al. 1998) removed assuming $A_{V} / E(B-V) \approx 3.1$ and the reddening

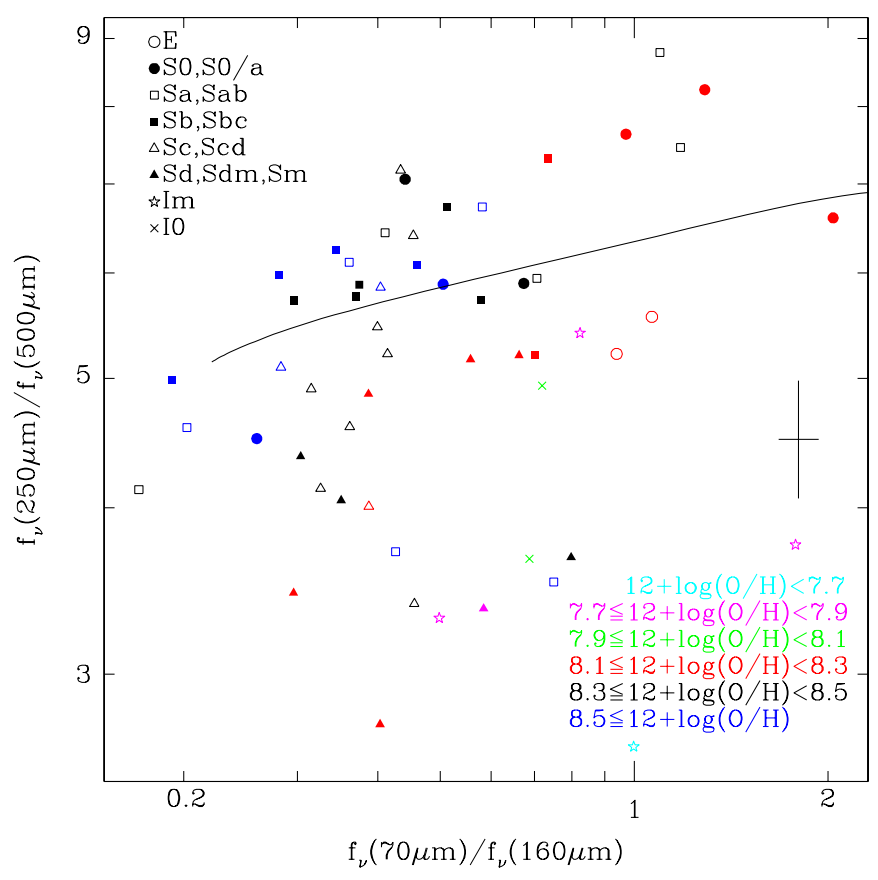

Figure 4. Second far-infrared/submillimeter color-color diagram for the KINGFISH sample (see also Figure 3).

curve of Li \& Draine (2001). ${ }^{23}$ No color corrections have been applied to the data in Table 2. The most recent calibrations are used for both SPIRE and PACS photometry, including the "FM, 6" PACS calibration which lowers the fluxes for extended sources by $10 \%-20 \%$ compared to the previous calibration, as described in the HIPE 7.0.0 documentation.

The superior sensitivity and angular resolution of Herschel enables a more detailed investigation of the faintest galaxies in our sample. For example, Dale et al. (2007) provide marginally significant MIPS flux densities at 70 and $160 \mu \mathrm{m}$ for NGC 1404 and DDO 165 , but they caution that the emission appearing within the apertures for these galaxies potentially derives from background galaxies. It is now clear based on the Herschel maps that these targets were indeed not detected by Spitzer (nor Herschel) at $\lambda \geqslant 70 \mu \mathrm{m}$.

The suite of far-infrared filter bandpasses available for Herschel and archival Spitzer data allows a direct comparison of the global flux densities measured for the SINGS and KINGFISH galaxy samples. The Spitzer and Herschel fluxes at 70 and $160 \mu \mathrm{m}$ are on average consistent; Figure 2 shows that the (error-weighted) ratios of Spitzer/Herschel flux densities agree fairly well, after accounting for differences in the Spitzer and Herschel spectral responses and calibration schemes. The agreement at $70 \mu \mathrm{m}$ is within $3 \%$ (with a $12 \%$ dispersion in the ratio), while at $160 \mu \mathrm{m}$ the MIPS flux densities are typically $6 \%$ larger (with a $16 \%$ dispersion). Galaxies fainter than $\sim 1$ Jy show a much larger dispersion in these ratios, but the flux densities for these targets are more susceptible to errors in sky estimation (Section 3.3).

Figures 3 and 4 provide a color-color snapshot of the Herschel global photometry. As expected, a clear correlation is seen in Figure 3 when the flux density ratios on both axes involve wavelengths that straddle the broad infrared peak of emission for most galaxies. Figure 4, on the other hand,

\footnotetext{
23 The corrections for Galactic extinction are very small at these wavelengths with the largest correction being $0.4 \%$ for IC 0342 at $70 \mu \mathrm{m}$, which lies at a Galactic latitude of $+10^{\circ}$ behind a foreground veil of $E(B-V) \sim 0.56 \mathrm{mag}$.
} 


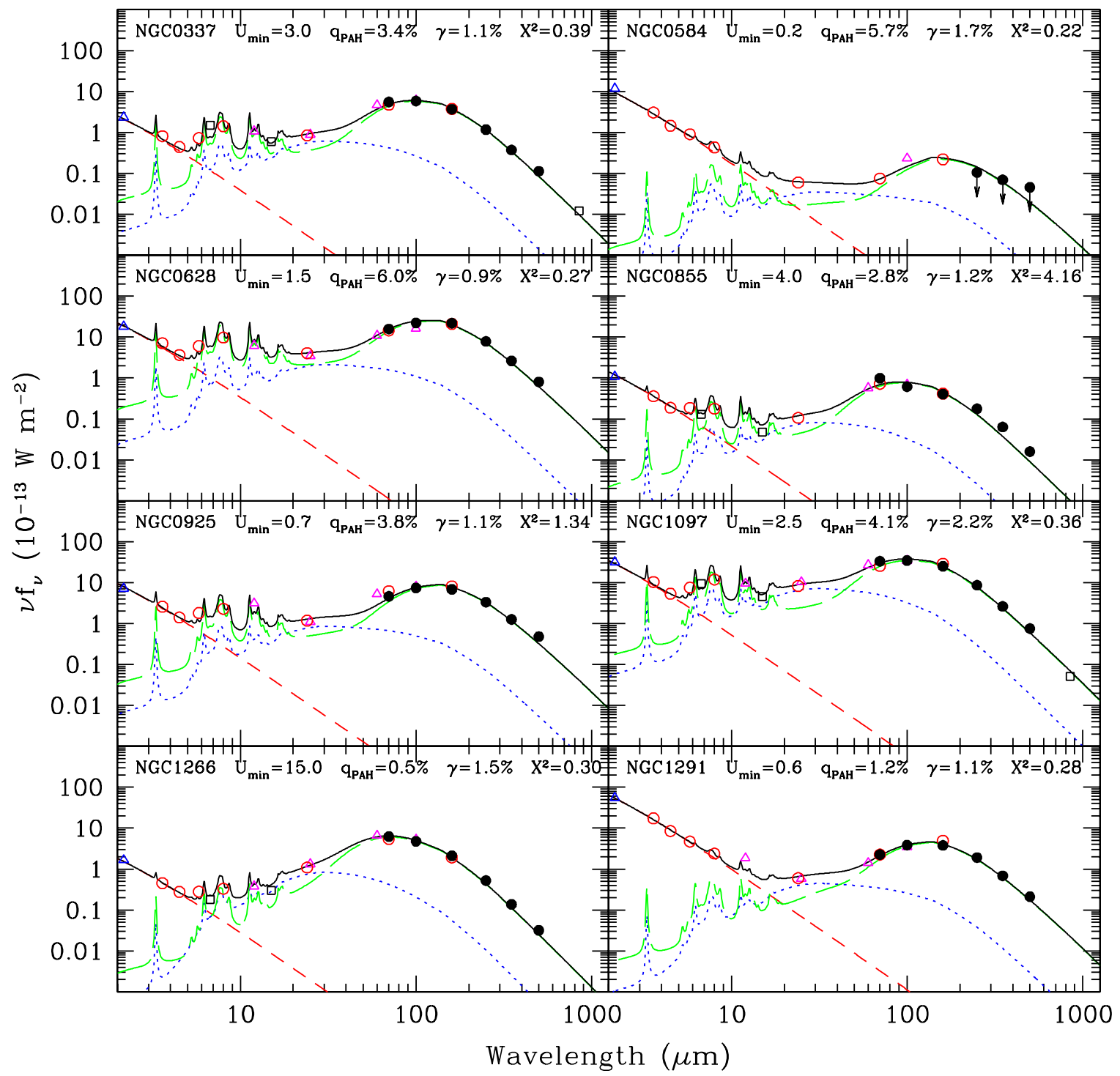

Figure 5. Globally integrated infrared/submillimeter spectral energy distributions for all the galaxies in the KINGFISH sample sorted by right ascension. Herschel data are represented by filled circles and ancillary data are indicated by open symbols (triangles: 2MASS and IRAS; circles: Spitzer; squares: ISO and SCUBA). Arrows indicate $5 \sigma$ upper limits. The solid curve is the sum of a $5000 \mathrm{~K}$ stellar blackbody (short dashed) along with models of dust emission from photodissociation regions (dotted; $U>U_{\min }$ ) and the diffuse ISM (long dashed; $U=U_{\min }$ ). The fitted parameters from these Draine \& $\mathrm{Li}$ (2007) model fits are listed within each panel along with the reduced $\chi^{2}$ (see Section 4.4 for details).

(A color version of this figure is available in the online journal.)

demonstrates that the galaxy spectral energy distributions in general do not form a simple one-parameter sequence. Galaxies are more complicated, with mixtures of dust temperatures and distributions of grain properties that vary from one galaxy to another. The galaxy types are fairly well distributed in terms of their infrared/submillimeter colors, though the Sc and Sd spirals tend to cluster toward cooler infrared colors (i.e., smaller values of $f_{v}(70 \mu \mathrm{m}) / f_{v}(160 \mu \mathrm{m}), f_{v}(70 \mu \mathrm{m}) / f_{v}(250 \mu \mathrm{m})$, and $\left.f_{v}(100 \mu \mathrm{m}) / f_{v}(500 \mu \mathrm{m})\right)$ and the magellanic irregulars (Type Im) have relatively large $500 \mu \mathrm{m}$ flux densities. This latter issue will be revisited in Section 4.6.

\subsection{The Observed Spectral Energy Distributions}

Figure 5 shows the observed infrared/submillimeter spectral energy distributions for the KINGFISH sample. Included in each panel, when available, are the Two Micron All Sky Survey
(2MASS) $K_{\mathrm{s}}$, ISO 6.75 and $15 \mu \mathrm{m}$, Spitzer 3.6, 4.5, 5.8, 8.0, 24, 70, and $160 \mu \mathrm{m}$, IRAS 12, 25, 60, and $100 \mu \mathrm{m}$, Herschel $70,100,160,250,350$, and $500 \mu \mathrm{m}$, and SCUBA 450 and $850 \mu \mathrm{m}$ band fluxes derived from this work and Dale et al. $(2007,2009)$. These data nominally reflect the global emission at each wavelength, but as pointed out in Draine et al. (2007), a subset of the SCUBA images suffers from various technical and observational issues. The data processing for scan-mapped SCUBA observations (NGC 4254, NGC 4579, and NGC 6946) removes an unknown contribution from extended emission; the areas mapped by SCUBA for NGC 1097, NGC 4321, and NGC 4736 were small and thus any errors in the large aperture corrections determined by Dale et al. (2007) for these three systems would have a significant impact on their inferred global fluxes; and the extra-nuclear submillimeter emission at $850 \mu \mathrm{m}$ is unreliably mapped in NGC 4594 due to contamination by an active galactic nucleus. Indeed, for all these special cases except 


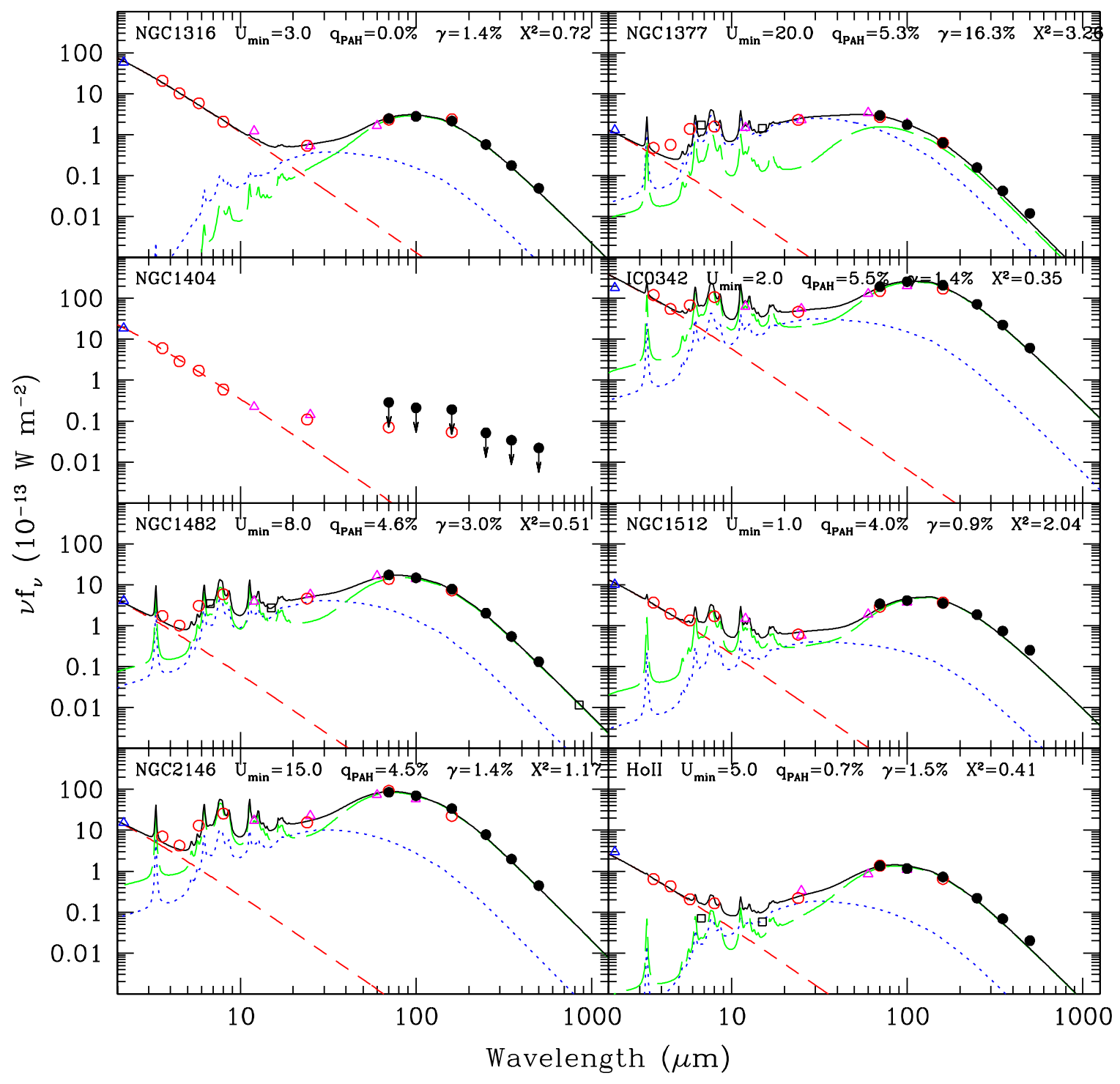

Figure 5. (Continued)

NGC 4736, the SCUBA data appear to fall appreciably below expectations based on extrapolations from the superior Herschel data.

\subsection{Fits to the Observed Spectral Energy Distributions}

To extract physical parameters from the broadband spectral data, the spectral energy distributions were fitted with the models of Draine \& $\mathrm{Li}$ (2007), models based on mixtures of amorphous silicate and graphitic dust grains that effectively reproduce the average Milky Way extinction curve and are consistent with observations of polycyclic aromatic hydrocarbon $(\mathrm{PAH})$ features and the variety of infrared continua in local galaxies. Draine \& $\mathrm{Li}(2007)$ use the size distributions of Weingartner \& Draine (2001) for dust in the diffuse Milky Way, except for adjustment of the parameters that characterize the PAH size distribution. The Draine \& Li (2007) dust models use the far-infrared and submillimeter opacities for graphite and amorphous silicate from Li \& Draine (2001). Li \& Draine (2001) used the graphite opacity from Draine \& Lee (1984), but made small modifications to the amorphous silicate opacity. The imagi- nary part of the amorphous silicate dielectric function $\epsilon_{2}(\lambda)$ was adjusted in order for the model to better match the average high Galactic latitude dust emission spectrum measured by COBE/Far-InfraRed Absolute Spectrophotometer (FIRAS) (Wright et al. 1991; Reach et al. 1995; Finkbeiner et al. 1999). The adjustments were modest: $\epsilon_{2}(\lambda)$ was unchanged for $\lambda<250 \mu \mathrm{m}$, and modified by less than $12 \%$ for $250 \mu \mathrm{m}<$ $\lambda<1100 \mu \mathrm{m}$. With this dielectric function for the amorphous silicate component, the Draine \& Li (2007) model gives generally good agreement with the observed submillimeter emission from the Milky Way diffuse ISM. Thus the Draine \& Li (2007) model has in effect been "tuned" to reproduce the diffuse emission from the local Milky Way. While the dust model used here is referenced as coming from Draine \& $\mathrm{Li}$ (2007), in fact two small changes have been incorporated since that publication: (1) there have been some small changes in some of the PAH band parameters, and (2) the graphite dielectric function has been modified to broaden out an opacity peak near $30 \mu \mathrm{m}$. These changes are described in G. Aniano et al. (2012, in preparation).

Building upon an idea put forth by Dale et al. (2001), Draine $\& \mathrm{Li}$ (2007) model interstellar dust heating within a galaxy with 


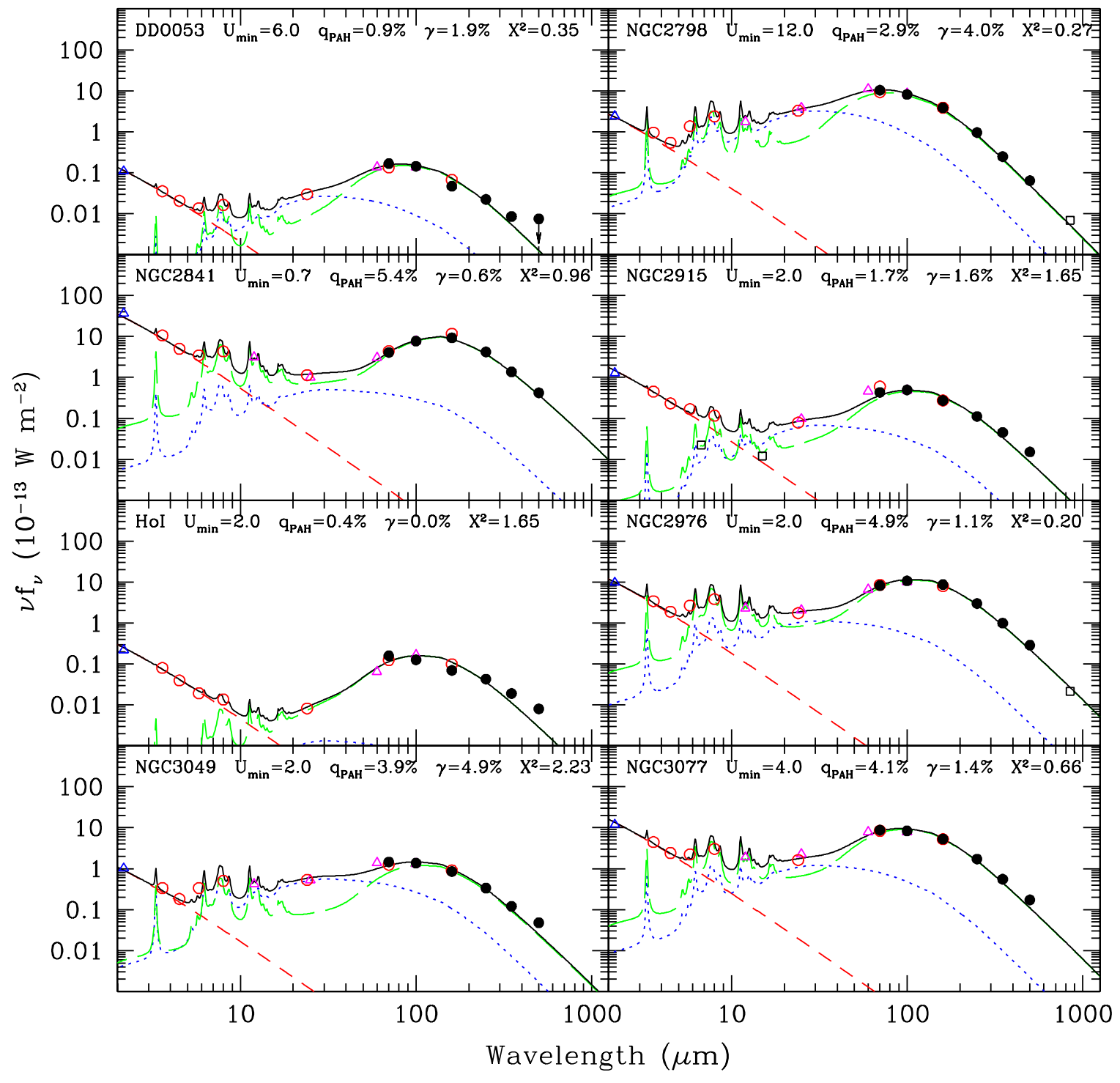

Figure 5. (Continued)

a $\delta$-function in interstellar radiation field intensity $U$ coupled with a power-law distribution over $U_{\min }<U<U_{\max }$,

$$
\begin{aligned}
d M_{\text {dust }} / d U= & M_{\text {dust }}\left[(1-\gamma) \delta\left(U-U_{\min }\right)\right. \\
& \left.+\gamma \frac{\alpha-1}{U_{\min }^{1-\alpha}-U_{\max }^{1-\alpha}} U^{-\alpha}\right]
\end{aligned}
$$

where $U$ is normalized to the local Galactic interstellar radiation field, $d M_{\text {dust }}$ is the differential dust mass heated by a range of starlight intensities $[U, U+d U], M_{\text {dust }}$ is the total dust mass, and $(1-\gamma)$ is the portion of the dust heated by the diffuse interstellar radiation field defined by $U=U_{\min }$. The minimum and maximum interstellar radiation field intensities span $0.01<U_{\min }<30$ and $3<\log U_{\max }<8$. See Section 5.5 of Dale et al. (2001) for a physical motivation of the powerlaw distribution in $U$, and Figure 4 of Draine \& $\mathrm{Li}$ (2007) for examples of translating $U$ to dust temperature for different grain sizes.

A sum of three different spectral energy distributions is fit to each galaxy: a blackbody of temperature $T_{*}=5000 \mathrm{~K}$, which Smith et al. (2007) find to be a good approximation to the stellar profile beyond $5 \mu \mathrm{m}$, along with two dust components. Following Draine et al. (2007), the sum can be expressed as

$$
\begin{aligned}
f_{v}^{\text {model }}= & \Omega_{*} B_{v}\left(T_{*}\right)+\frac{M_{\mathrm{dust}}}{4 \pi D^{2}}\left[(1-\gamma) p_{v}^{(0)}\left(q_{\mathrm{PAH}}, U_{\mathrm{min}}\right)\right. \\
& \left.+\gamma p_{v}\left(q_{\mathrm{PAH}}, U_{\min }, U_{\max }, \alpha\right)\right],
\end{aligned}
$$

where $\Omega_{*}$ is the solid angle subtended by stellar photospheres, $D$ is the distance to the galaxy, and $\gamma$ and $(1-\gamma)$ are the fractions of the dust mass heated by the "power-law" and "deltafunction" starlight distributions, respectively. $p_{v}^{(0)}\left(q_{\mathrm{PAH}}, U_{\min }\right)$ and $p_{v}\left(q_{\mathrm{PAH}}, U_{\min }, U_{\max }, \alpha\right)$ are, respectively, the emitted power per unit frequency per unit dust mass for dust heated by a single starlight intensity $U_{\min }$ and dust heated by a power-law distribution of starlight intensities $d M / d U \propto U^{-\alpha}$ extending from $U_{\min }$ to $U_{\max }$. The $U=U_{\min }$ component may be interpreted as the dust in the general diffuse ISM. The power-law starlight distribution allows for dust heated by more intense starlight, such as in the intense photodissociation regions (PDRs) in starforming regions. For simplicity, emission from dust heated by $U>U_{\min }$ will be referred to as the "PDR" component, and the emission from dust heated by $U=U_{\min }$ will be referred to as the "diffuse ISM" component. Finally, the fractional contribution by 


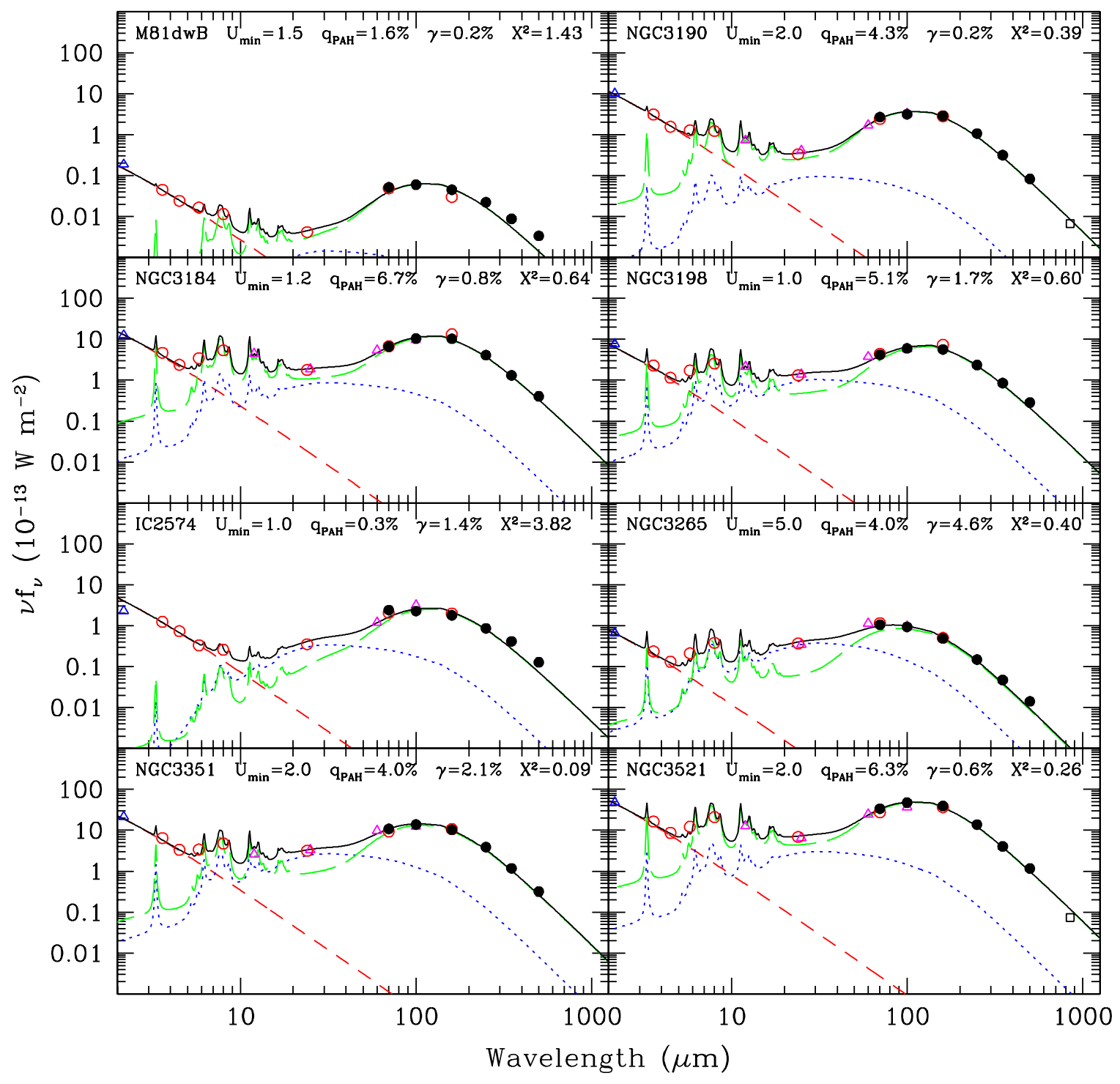

Figure 5. (Continued)

total dust mass from PAHs, denoted $q_{\mathrm{PAH}}$, varies between $0 \%$ and $12 \%$ with a model grid spacing of $0.1 \%$ in $q_{\mathrm{PAH}}$.

Draine et al. (2007) find that fits to the (SINGS) global spectral energy distributions of nearby galaxies are insensitive to the minimum radiation field intensity, the maximum radiation field intensity, and the power-law parameter $\alpha$. We adopt their choice to fix $U_{\max }=10^{6}$ and $\alpha=2$ to minimize the number of free parameters. Draine et al. (2007) use a minimum value of 0.7 for $U_{\min }$, but we choose to extend this range down to 0.01 due to the availability of SPIRE data longward of $160 \mu \mathrm{m}$ and the resulting potential for having detected very cold dust emission. The free parameters $\Omega_{*}, M_{\text {dust }}, q_{\mathrm{PAH}}, U_{\mathrm{min}}$, and $\gamma$ are found via $\chi^{2}$ minimization:

$$
\chi^{2}=\sum_{b} \frac{\left(f_{\nu, b}^{\mathrm{obs}}-f_{\nu, b}^{\text {model }}\right)^{2}}{\left(\sigma_{b}^{\mathrm{obs}}\right)^{2}+\left(\sigma_{b}^{\text {model }}\right)^{2}}
$$

where $f_{v, b}^{\text {model }}$ is the model flux density obtained after convolving the model with the $b$ filter bandpass, $\sigma_{b}^{\text {obs }}$ is the uncertainty in the observed flux density, and $\sigma_{b}^{\text {model }}$ is set to $0.1 f_{v, b}^{\text {model }}$ to allow for the uncertainty intrinsic to the model.
Figure 5 displays the fits of the Draine \& Li (2007) models to the combined broadband observations from the Spitzer and Herschel observatories. The median reduced chi-squared value is near unity $(\sim 0.7)$, and with just a few exceptions the fits are quite reasonable. The most challenging spectral energy distributions to fit involve spatially variable Milky Way cirrus coupled with a faint target, and thus any errors in determining the value of the local sky has a relatively large impact on the inferred fluxes (i.e., dwarf galaxies such as DDO 053, M81 dwarf B, and the faint elliptical NGC 0584).

A wealth of information can be extracted from such fits. Figure 6, for example, uses these fits to provide a glimpse into how the global spectral energy distributions depend on the star formation rate and total infrared luminosity. The infrared spectral energy distributions typically peak at shorter wavelengths for KINGFISH galaxies with higher star formation rates and infrared luminosities. There are exceptions to these generalizations, however, especially for lower luminosity systems. A full tabulation of the output parameters for the KINGFISH sample will be presented in G. Aniano et al. (2012, in preparation). Here we restrict our analysis of the output parameters to (1) evaluating the impact of including the Herschel data in these 


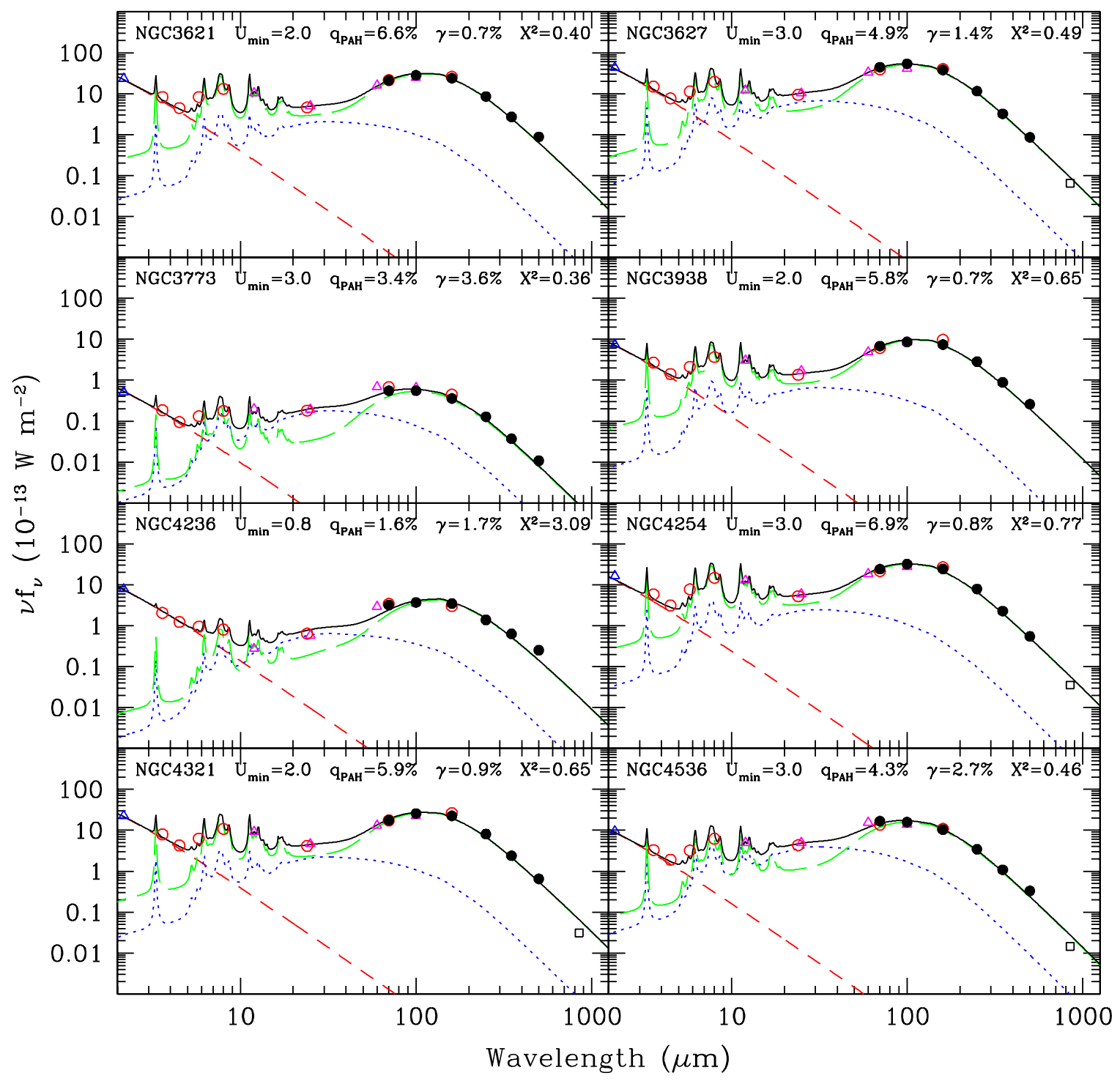

Figure 5. (Continued)

fits, and (2) comparing the dust masses found through Draine \& Li (2007) fits with those from single-temperature modified blackbody fits.

\subsection{Spectral Energy Distribution Fit Parameters}

Figure 7 compares (ratios of) the output parameters $\gamma, q_{\mathrm{PAH}}$, $U_{\min }$, and $M_{\text {dust }}$ when the fits are executed with and without the inclusion of Herschel photometry. All four parameters are relatively unchanged, on average, when Herschel broadband data are added to those from Spitzer. The largest average deviation in the ratio from unity is seen in the top panel, where the fraction of dust heated by PDRs found by using both Spitzer and Herschel data is on average $(21 \pm 4) \%$ larger than that using just Spitzer data. Interestingly, the largest dispersions in the distributions in Figure 7 are for $U_{\min }$ and $M_{\text {dust }}$, indicating the importance of Herschel data in assessing these parameters. In addition, all four parameters show ratio distributions that are fairly evenly distributed about their means, though the scatter shrinks for cooler galaxies. At first blush it may be surprising that the inclusion of Herschel far-infrared/submillimeter has any impact on a parameter such as $q_{\mathrm{PAH}}$ that is sensitive to mid-infrared PAH features, but recall that $q_{\mathrm{PAH}}$ is the PAH mass abundance with respect to the total dust mass, and clearly Herschel photometry has an important role in determining the latter. Finally, even though $U_{\min }$ was allowed to go as low as 0.01 , the smallest fitted value using the combined Spitzer and Herschel data sets is 0.6 , similar to the $U_{\min }$ floor advocated by Draine et al. (2007).

Figure 8 shows the same ratios plotted as a function of oxygen abundance (Moustakas et al. 2010). The dependence of KINGFISH dust masses on metallicity are consistent with those found by Galametz et al. (2011) in a study of 52 galaxies with submillimeter data: dust masses computed for metal-rich (metalpoor) galaxies are smaller (larger) when submillimeter data are included in the fit. Galametz et al. (2011) argue that most metalrich galaxies have their dust emission peak in the far-infrared beyond $160 \mu \mathrm{m}$, and that submillimeter data are required to fine-tune dust measures for such systems. KINGFISH galaxies with oxygen abundances $12+\log (\mathrm{O} / \mathrm{H})$ greater (less) than 8.1 on the empirical Pilyugin \& Thuan (2005) metallicity scale are computed to have an average of $0.06 \pm 0.03$ dex less $(0.28 \pm 0.09$ dex more $)$ dust mass when submillimeter data are used. This demarcation in metal abundance is similar to 


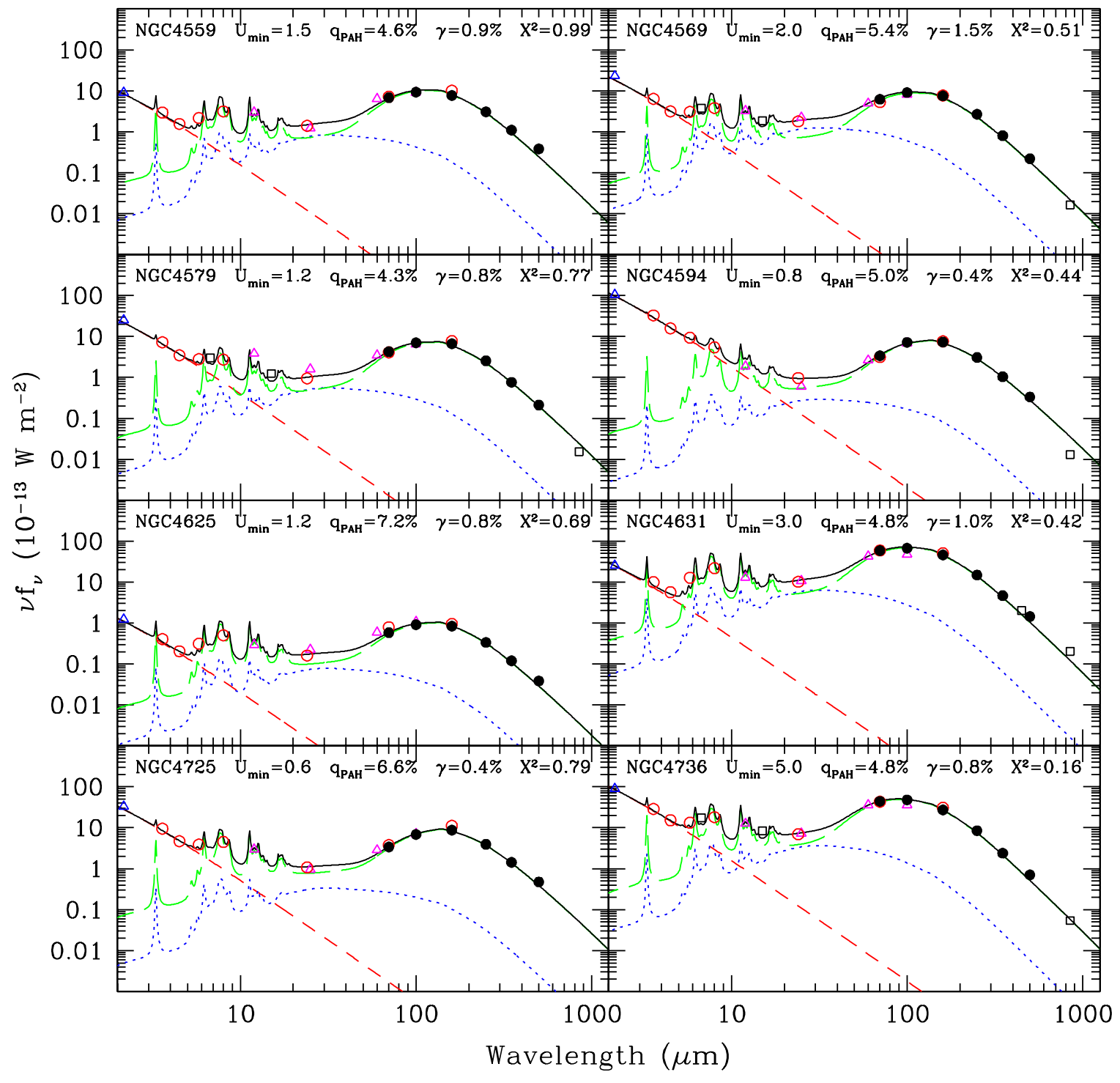

Figure 5. (Continued)

that studied by others in quantifying, for example, the relative importance of PAH emission in galaxies (e.g., Hunt et al. 2005; Draine et al. 2007; Smith et al. 2007; Engelbracht et al. 2008). In short, perhaps KINGFISH data show metallicity-dependent dust mass trends similar to those found by Galametz et al. (2011), but it would be useful to have more data to confirm any such trend.

\subsection{Comparison with Dust Masses from Blackbody Fits}

Galaxy dust masses are typically estimated by fitting a single modified blackbody to a selection of infrared/submillimeter continuum fluxes,

$$
M_{\mathrm{dust}}=\frac{f_{v} D^{2}}{\kappa\left(v_{0}\right) B_{v}\left(T_{1}\right)}\left(\frac{v_{0}}{v}\right)^{\beta_{1}}
$$

or in some cases a superposition of two modified blackbodies of two different dust temperatures,

$M_{\text {dust }}=\frac{f_{\nu} D^{2}}{\kappa\left(v_{0}\right)}\left[x B_{v}\left(T_{1}\right)\left(\frac{v}{v_{0}}\right)^{\beta_{1}}+(1-x) B_{v}\left(T_{2}\right)\left(\frac{v}{v_{0}}\right)^{\beta_{2}}\right]^{-1}$, where $\kappa\left(v_{0}\right)$ is the dust mass absorption coefficient at the reference frequency $\nu_{0}, T_{1}$ and $T_{2}$ are the modeled dust temperatures, $\beta_{1}$ and $\beta_{2}$ are the dust emissivity indices, and $0<x<1$; some authors choose to fix the dust emissivity index(indices) (e.g., Dunne \& Eales 2001; Kovács 2006; Pascale et al. 2009). While such approaches provide quick and simple routes to gauging the dust mass, they do not capture the full range of dust temperatures inherent to any galaxy. However, due to their popularity it is instructive to compare blackbody-based dust masses to those determined from more nuanced models.

Figure 9 compares the dust masses obtained by using a singletemperature modified blackbody (Equation (7)) to those obtained in Section 4.4. Both approaches utilize the results of $\mathrm{Li}$ $\&$ Draine (2001) for dust absorption cross sections, and in particular $v_{0}=c / 250 \mu \mathrm{m}=1.20 \mathrm{THz}$ and $\kappa\left(v_{0}\right) \approx 0.48 \mathrm{~m}^{2} \mathrm{~kg}^{-1}$ are used for the SPIRE $250 \mu \mathrm{m}$ band in determining the (modified) blackbody dust mass. In addition, both the dust temperature $T_{\mathrm{d}}$ and the dust opacity coefficient $\beta$ are allowed to freely vary in the fit for each galaxy; the ranges for the fitted values are quite reasonable given that KINGFISH does not contain any extreme objects: $18 \lesssim T_{\mathrm{d}} \lesssim 40 \mathrm{~K}$ and $1.2 \lesssim \beta \lesssim 1.9$ (Figure 10; see Skibba et al. 2011 for similar results based on 


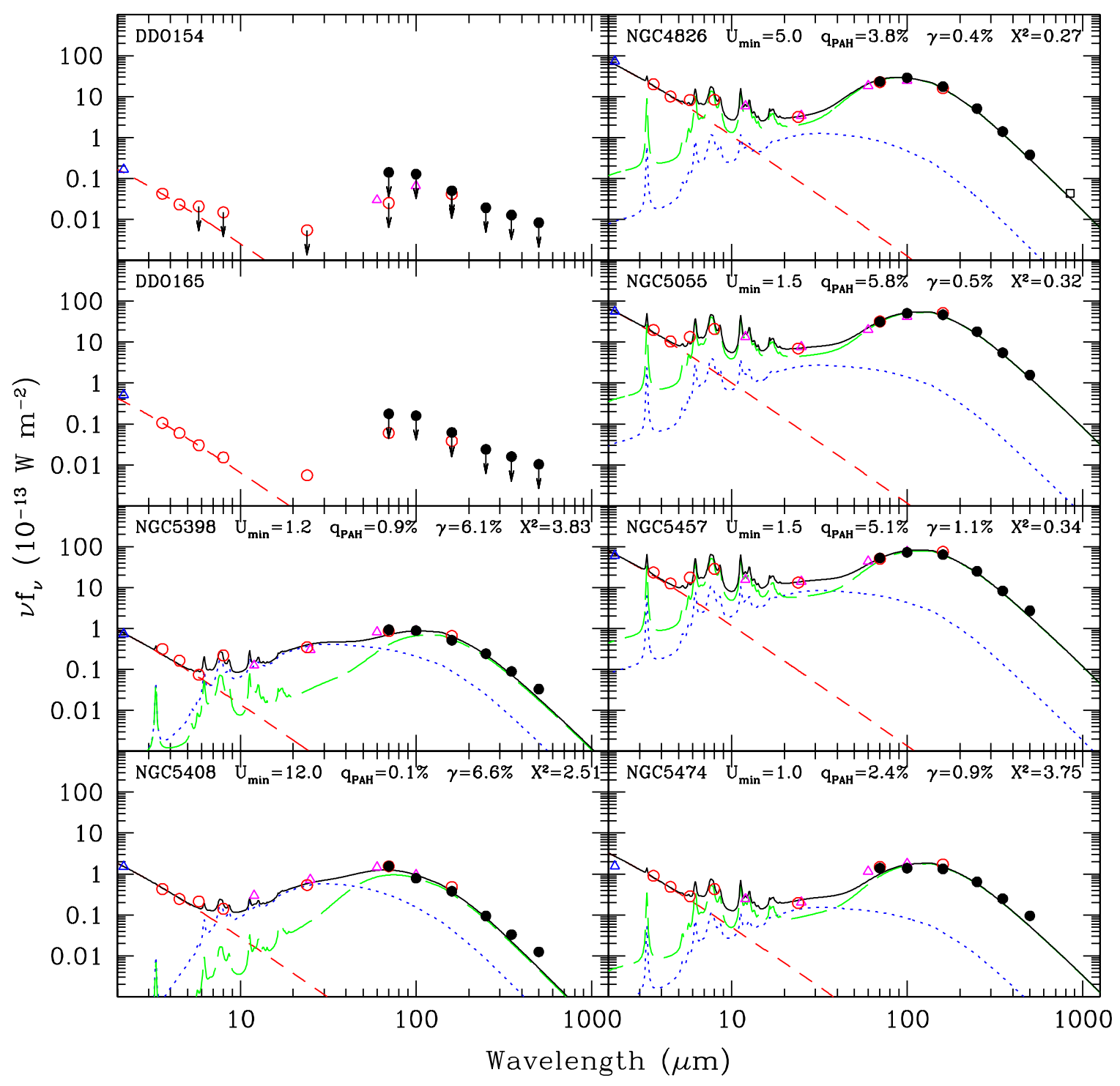

Figure 5. (Continued)

modified blackbody fits to KINGFISH targets). In order to avoid contributions from stochastically heated dust grains in the computation of the blackbody-based dust masses, the top panel of Figure 9 shows results when only Herschel photometric bands from $100 \mu \mathrm{m}$ through $500 \mu \mathrm{m}$ are included in the fits. Results are not significantly different when $70 \mu \mathrm{m}$ data are included (bottom panel); the median ratio in the top (bottom) panel is $0.53(0.46)$. Figure 9 indicates that single-temperature (modified) blackbody dust masses typically underestimate the values obtained through a Draine \& Li (2007) formalism by nearly a factor of two $(\sim 1.9)$, and there is a trend toward larger underestimates for galaxies exhibiting cooler far-infrared colors.

Similar results are obtained after fixing $\beta$ to either 1.5 or 2.0, except for the situation where the blackbody fits are carried out over the wider 70-500 $\mu \mathrm{m}$ wavelength baseline for $\beta=2.0$. In that case the fitted dust temperatures are lower in order to compensate for an overly steep emissivity dependence on wavelength, resulting in larger quantities of dust and only a $25 \%$ underestimate in the dust mass compared to those obtained from Draine \& Li (2007), echoing the findings in Magrini et al. (2011). Dunne \& Eales (2001) likewise find a factor of two deficiency for single blackbody-based dust masses, in their case compared to the dust mass derived from two (modified) blackbodies (see also Skibba et al. 2011). Figure 11 shows a primary reason for the discrepancy: even when limited to $\lambda \geqslant 100 \mu \mathrm{m}$ photometry, single-temperature blackbody fits overestimate the dust temperature, thus underestimating the dust mass. The single-temperature model does not account for the contribution of warm dust emitting at shorter wavelengths and the temperatures are driven toward higher values in the attempt to fit both the short- and long-wavelength far-infrared emission. This effect is accentuated for galaxies with cooler large dust grains whose emission peaks at longer infrared wavelengths. A more comprehensive and detailed comparison of various dust mass indicators is studied in K. D. Gordon et al. (2012b, in preparation).

\subsection{Submillimeter Excess}

As described in Section 1, several studies of dwarf galaxies show significant excess emission at submillimeter wavelengths. Inspection of Figure 5 shows that only a few spiral and ellipti- 


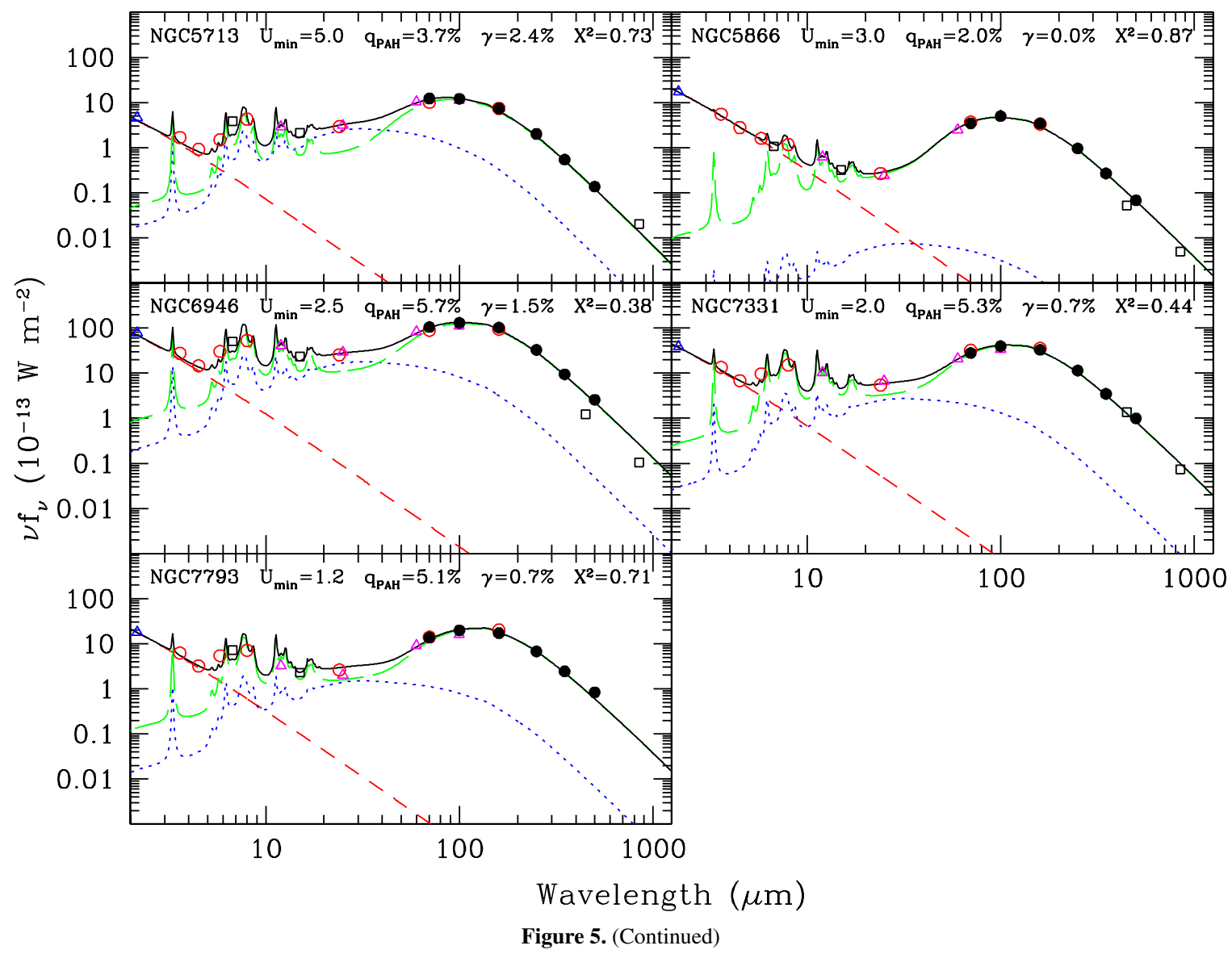

cal KINGFISH galaxies have $500 \mu \mathrm{m}$ fluxes that are noticeably above the fitted model curves (e.g., NGC 3049 and NGC 5474). However, it is interesting that dwarf/irregular/Magellanic galaxies preferentially show this excess. There are a total of 12 KINGFISH galaxies of Type Im (Magellanic irregular), Type I0 (non-Magellanic irregular), or Type Sm (Magellanic spiral), and of the nine with detections at $500 \mu \mathrm{m}$, eight show hints of $500 \mu \mathrm{m}$ emission above the Draine \& Li (2007) model fit (IC 2574, Holmberg I, Holmberg II, M81 dwarf B, NGC 2915, NGC 4236, NGC 5398, and NGC 5408; see also Figure 3). Quantitatively, the observed $500 \mu \mathrm{m}$ excess can be defined with respect to the model prediction at $500 \mu \mathrm{m}$, namely,

$$
\xi(500 \mu \mathrm{m})=\frac{v f_{v}(500 \mu \mathrm{m})_{\text {observed }}-v f_{v}(500 \mu \mathrm{m})_{\text {model }}}{v f_{v}(500 \mu \mathrm{m})_{\text {model }}} .
$$

A dozen KINGFISH galaxies show $\xi(500 \mu \mathrm{m})>0.6$, including all eight of the dwarf/irregular/Magellanic galaxies listed above. However, it should be noted that interpreting $\xi(500 \mu \mathrm{m})$ for these systems is complicated by the fact that they are typically faint in the far-infrared/submillimeter (e.g., Walter et al. 2007) and thus their measured flux values are the least reliable. Nonetheless, it is interesting that the lowest metallicity objects in the KINGFISH sample are the sources most likely to show a submillimeter excess and thus potentially harbor the coldest dust or have peculiar dust grain characteristics. A detailed analysis of the submillimeter excess in KINGFISH galaxies is being carried out by Galametz et al. (2011), K. D. Gordon et al. (2012a, in preparation), and L. Hunt et al. (2012, in preparation).

\section{DISCUSSION AND SUMMARY}

Spatially integrated far-infrared and submillimeter flux densities from the Herschel Space Observatory are provided for the 61 objects in the KINGFISH sample of nearby galaxies. All but three galaxies are detected in the far-infrared by PACS and all but four galaxies are detected in the submillimeter by SPIRE. The (color-corrected) Herschel PACS $70 \mu \mathrm{m}$ global flux densities agree with Spitzer MIPS $70 \mu \mathrm{m}$ counterparts to within $3 \%$ (with a $12 \%$ dispersion) on average; the MIPS $160 \mu \mathrm{m}$ flux densities are typically 6\% larger than the PACS $160 \mu \mathrm{m}$ flux densities (with a $16 \%$ dispersion).

The dust emission models described in Draine \& Li (2007) and Draine et al. (2007) are fit to the combined Spitzer and Herschel 3.6-500 $\mu \mathrm{m}$ data set. The fits provide constraints on the total dust mass $M_{\text {dust }}$, the PAH mass fraction $q_{\mathrm{PAH}}$, and the characteristics of the radiation fields that heat the dust including the fraction $\gamma$ of the dust mass that is located in regions with $U>U_{\min }$, and the complementary fraction $1-\gamma$ that is located in the general diffuse ISM. A full tabulation of the fit parameters will be presented in G. Aniano et al. (2012, in preparation); analysis of the fit results here is restricted to comparisons between fits with and without inclusion of the Herschel data. In general, the fits to Spitzer+Herschel data produce parameter values that are consistent, to within a factor of two, with those when just Spitzer data are fitted. However, the KINGFISH galaxies with oxygen abundances less than $12+\log (\mathrm{O} / \mathrm{H}) \lesssim 8$ tend to show larger dust masses and smaller PAH mass fractions when SPIRE data are included in the fits. A similar characteristic oxygen abundance has been noted in other studies of the PAH abundance in galaxies (e.g., Hunt et al. 2005; Draine et al. 2007; 


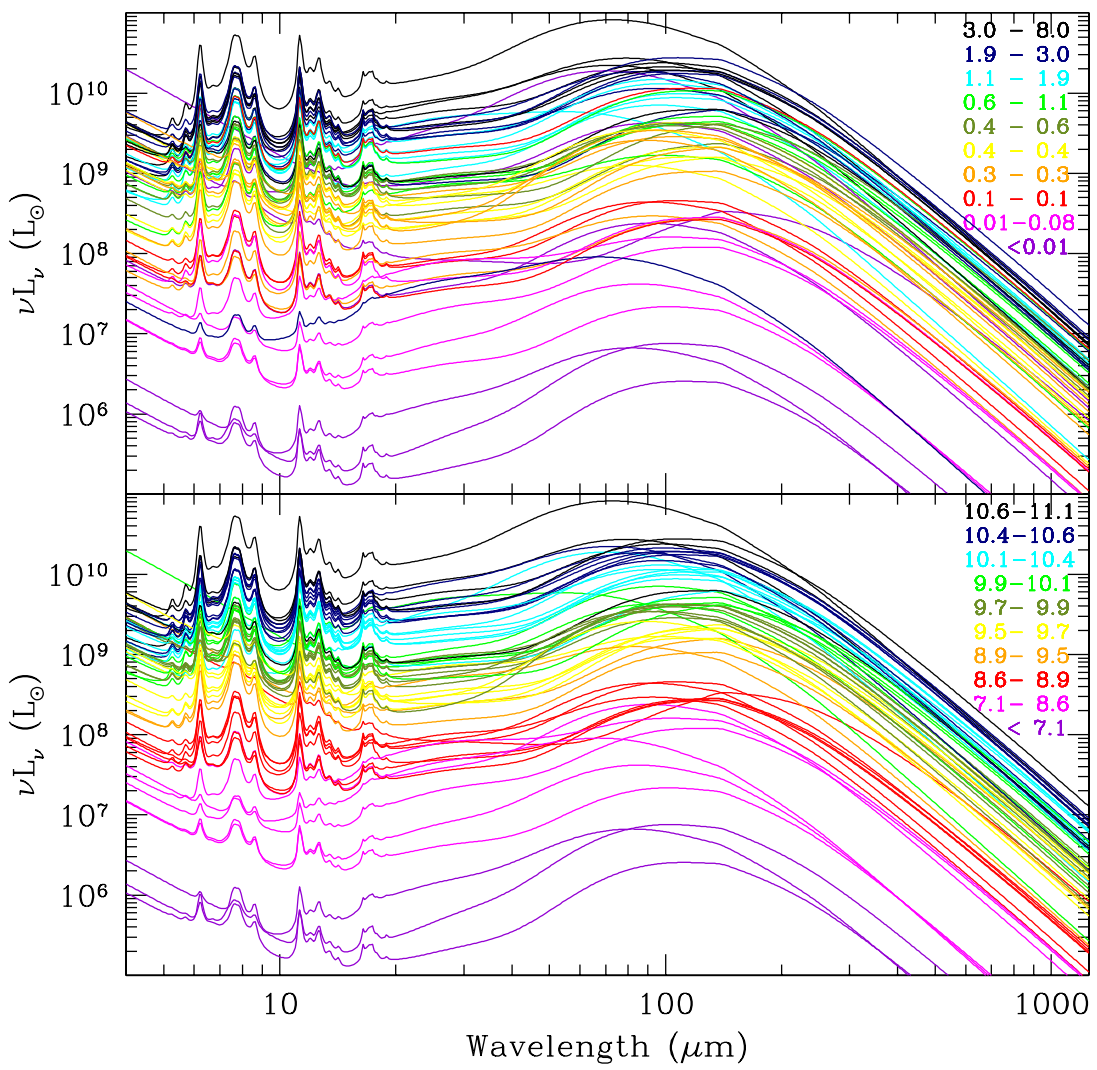

Figure 6. Collection of Draine \& $\mathrm{Li}$ (2007) model fits, color coded according to star formation rate in $M_{\odot} \mathrm{yr}^{-1}$ in the top panel (Kennicutt et al. 2012) and $\log _{10}\left(L_{\mathrm{TIR}} / L_{\odot}\right)$ in the bottom panel (see Table 1$)$.

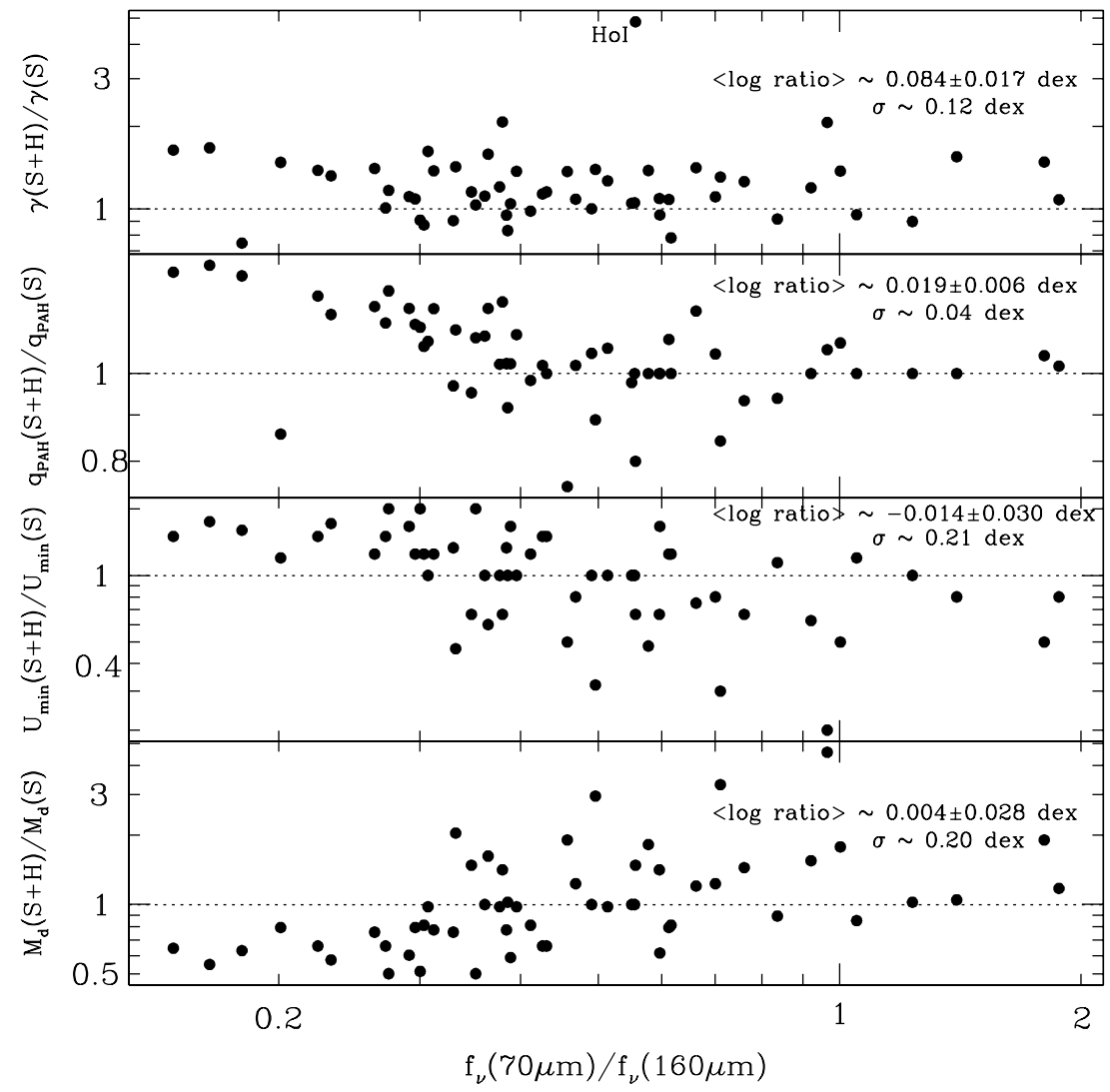

Figure 7. Comparison of the dust model parameters obtained from fitting Draine \& Li (2007) spectral energy distribution models to the entire observed photometric data from 3.6 to $500 \mu \mathrm{m}$ for fits including both Spitzer and Herschel data ("S+H") vs. fits using just the Spitzer photometry ("S"). The parameters constrain the quantity of interstellar dust and their heating; see Section 4.3. The comparison is made as a function of the $f_{v}(70 \mu \mathrm{m}) / f_{v}(160 \mu \mathrm{m})$ ratio, which is related to the average temperature of the interstellar dust grains. Reference dotted lines are drawn for a ratio of unity. 


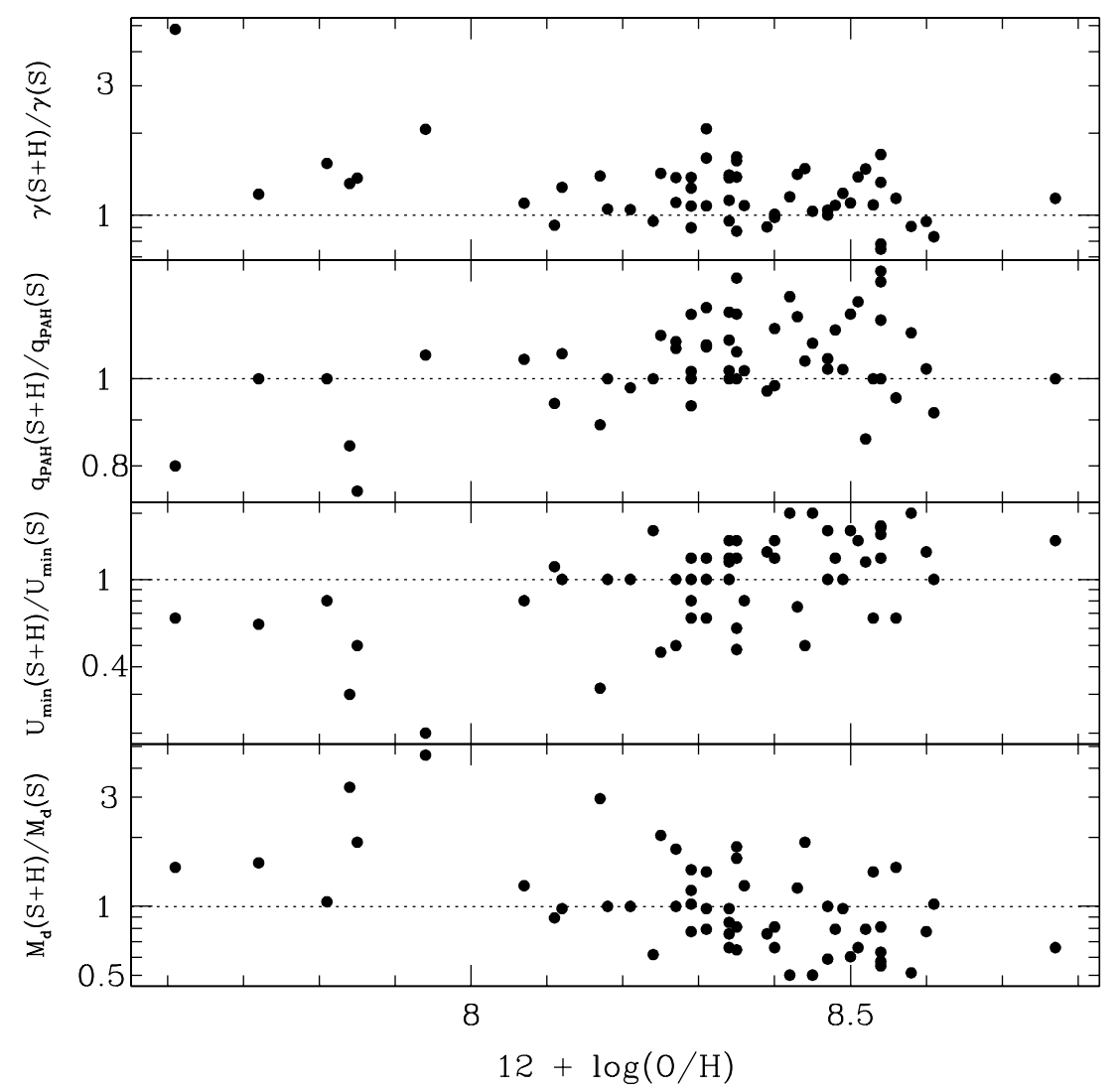

Figure 8. Same as Figure 7 except plotted as a function of oxygen abundance as presented in Moustakas et al. (2010) using data placed on the Pilyugin \& Thuan (2005) metallicity scale.

Smith et al. 2007; Engelbracht et al. 2008). In addition, the fraction of the dust mass located in regions with $U>U_{\min }, \gamma$, is $(21 \pm 4) \%$ larger when Herschel data are included in the fits. For $\alpha=2$ the fraction of the total dust luminosity contributed by regions with $U>100$ is given by Equation (29) of Draine \& $\operatorname{Li}(2007)$ :

$$
\begin{aligned}
& f\left(L_{\text {dust }}, U>10^{2}\right) \\
& \quad=\frac{\gamma \ln \left(U_{\max } / 10^{2}\right)}{(1-\gamma)\left(1-U_{\min } / U_{\max }\right)+\gamma \ln \left(U_{\max } / U_{\min }\right)} .
\end{aligned}
$$

This parameter is $(16 \pm 4) \%$ larger when Herschel data are included in the fits. These subtle differences in the fits presumably reflect the unprecedented ability of Herschel to properly account for contributions from cold dust grains, grains that sustain their meager thermal emission through heating by the diffuse radiation field that permeates a galaxy's ISM.

The presence of an excess of emission in the submillimeter has been noted in the literature, particularly for low-metallicity galaxies. Most KINGFISH galaxies are well modeled by spectral energy distributions consistent with emission curves from the Milky Way and nearby galaxies without needing to invoke an additional cold dust component. However, eight of the nine $\mathrm{dwarf} /$ irregular/Magellanic galaxies with detections at $500 \mu \mathrm{m}$ show evidence for significant excess of emission at this wavelength, at least with respect to the expectations based on the Draine \& Li (2007) model fits. These excesses, in fact, are the reason their dust masses are larger when Herschel data are included in the fits described above, assuming these excesses are attributable to increased quantities of very cold dust. It is unclear

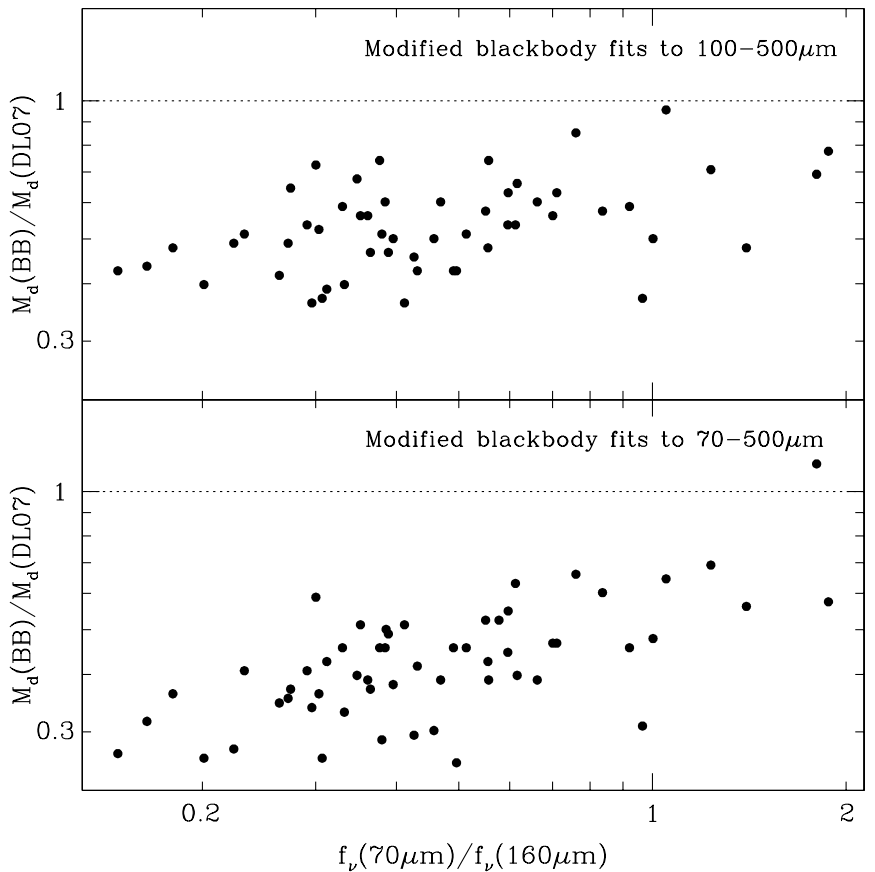

Figure 9. Ratio of single-temperature, blackbody-based dust mass to that obtained from Draine \& Li (2007) model fits to the observed infrared/submillimeter spectral energy distributions. Top (Bottom): the modified blackbody fits are based on the (error-weighted) Herschel 100, 160, 250, 350 , and $500 \mu \mathrm{m}(70,100,160,250,350$, and $500 \mu \mathrm{m})$ photometry, and both $T_{\mathrm{d}}$ and the dust opacity coefficient $\beta$ are allowed to vary freely. Reference dotted lines are drawn for a ratio of unity. 


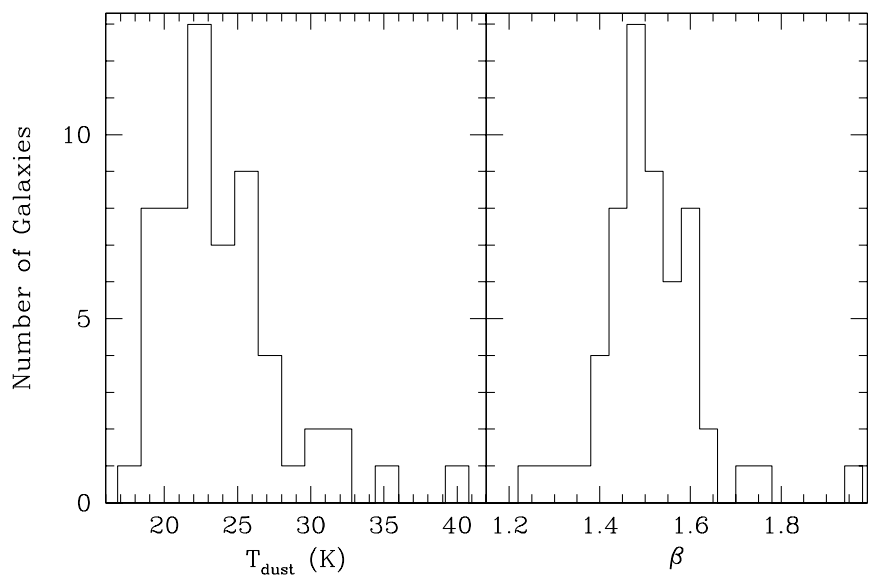

Figure 10. Distributions of dust temperature and dust emissivity index $\beta$ when single-temperature modified blackbodies are fit to the $(100-500 \mu \mathrm{m})$ infrared spectral energy distributions.

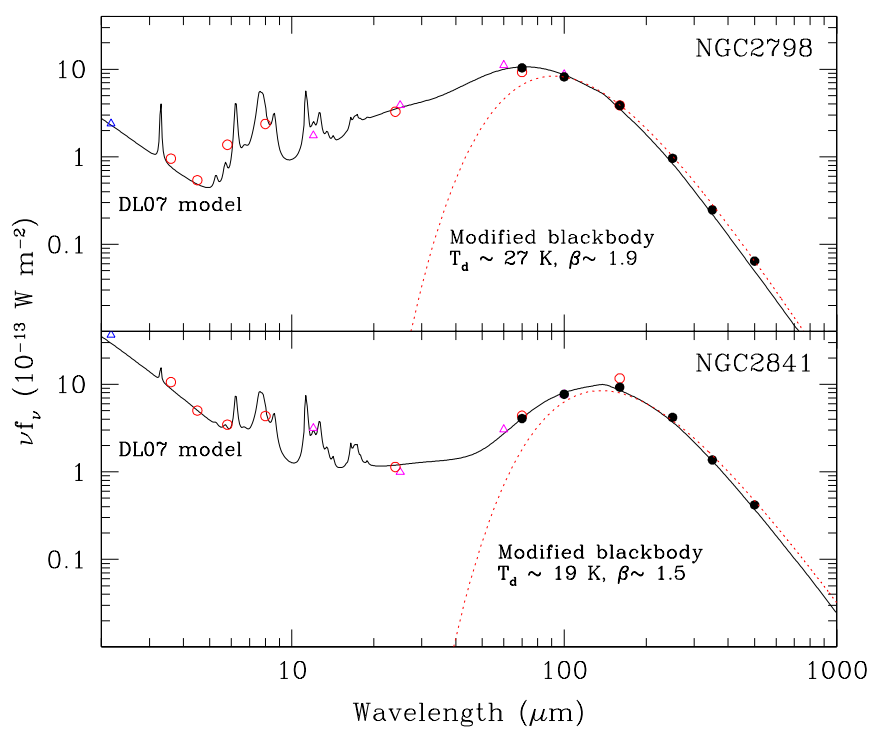

Figure 11. Comparison of fitting Draine \& Li (2007) models to 3.6-500 $\mu \mathrm{m}$ data vs. fits of single-temperature blackbodies to $100-500 \mu \mathrm{m}$ photometry for a galaxy with warm dust (NGC 2798) and a galaxy with cool dust (NGC 2841). (A color version of this figure is available in the online journal.)

why low-metallicity dwarf irregular galaxies exhibit a propensity for conspicuous cold dust emission. In fact, their spectral energy distributions do not typically peak at longer wavelengths than is seen for the more metal-rich galaxies; the KINGFISH dwarf galaxies are not colder than average, they simply show $500 \mu \mathrm{m}$ excesses. Perhaps such environments promote unusual dust emissivities that lead to the observed excesses (see also Bot et al. 2010 for additional explanations).

It is commonplace to find in the literature dust masses based on fits to single modified blackbody profiles, with the dust temperature and dust emissivity modifier $v^{\beta}$ serving as potential free parameters. Blackbody-based dust masses are on average a factor of $\sim 1.9$ smaller than those obtained through fits of Draine \& Li (2007) models, and the disagreement is larger for galaxies with cooler far-infrared colors. This systematic difference is due to the superior ability of the Draine \& Li (2007) dust model to represent the dust emission spectrum from the near-infrared through the submillimeter, with (for a given value of $q_{\mathrm{PAH}}$ ) a single dust opacity function $\kappa_{v}$, but allowing for a distribution of starlight heating intensities and resulting dust temperature distributions.

Herschel is an ESA space observatory with science instruments provided by European-led Principal Investigator consortia and with important participation from NASA. IRAF, the Image Reduction and Analysis Facility, has been developed by the National Optical Astronomy Observatories and the Space Telescope Science Institute.

\section{REFERENCES}

Ade, P. A. R., Aghanim, N., Arnaud, M., et al. 2011a, A\&A, 536, A17 Ade, P. A. R., Aghanim, N., Arnaud, M., et al. 2011b, A\&A, 536, A16 Aguirre, J. E., Bezaire, J. J., Cheng, E. S., et al. 2003, ApJ, 596, 273

Benford, D. J., Cox, P., Omont, A., Phillips, T. G., \& McMahon, R. G. 1999, ApJ, 518,65

Bolatto, A. D., Jackson, J. M., Wilson, C. D., \& Moriarty-Schieven, G. 2000, ApJ, 532, 909

Boselli, A., Eales, S., Cortese, L., et al. 2010, PASP, 122, 261

Bot, C., Ysard, N., Paradis, D., et al. 2010, A\&A, 523, 20

Dale, D. A., Cohen, S. A., Johnson, L. C., et al. 2009, ApJ, 703, 517

Dale, D. A., Gil de Paz, A., Gordon, K. D., et al. 2007, ApJ, 655, 863

Dale, D. A., \& Helou, G. 2002, ApJ, 576, 159

Dale, D. A., Helou, G., Contursi, A., Silbermann, N. A., \& Kolhatkar, S. 2001, ApJ, 549, 215

Diolaiti, E., Bendinelli, O., Bonaccini, D., et al. 2000, A\&A, 147, 335

Draine, B. T., Dale, D. A., Bendo, G., et al. 2007, ApJ, 663, 866

Draine, B. T., \& Lee, H. M. 1984, ApJ, 285, 89

Draine, B. T., \& Li, A. 2007, ApJ, 657, 810

Dunne, L., \& Eales, S. 2001, MNRAS, 327, 697

Dupac, X., Bernard, J.-P., Boudet, N., et al. 2003, A\&A, 404, 11

Engelbracht, C. W., Hunt, L. K., Skibba, R. A., et al. 2010, A\&A, 518, L56 Engelbracht, C. W., Rieke, G. H., Gordon, K. D., et al. 2008, ApJ, 678, 804

Finkbeiner, D. P., Davis, M., \& Schlegel, D. J. 1999, ApJ, 524, 867

Galametz, M., Madden, S., Galliano, F., et al. 2009, A\&A, 508, 645

Galametz, M., Madden, S. C., Galliano, F., et al. 2011, A\&A, 532, 56

Galliano, F., Hony, S., Bernard, J.-P., et al. 2011, A\&A, 536, 88

Galliano, F., Madden, S., Jones, A., Wilson, C., \& Bernard, J.-P. 2005, A\&A, 434,867

Gordon, K. D., Galliano, F., Hony, S., et al. 2010, A\&A, 518, L89

Griffin, M. J., Abergel, A., Abreu, A., et al. 2010, A\&A, 518, L3

Hunt, L., Bianch, S., \& Maiolino, R. 2005, A\&A, 434, 849

Kennicutt, R. C., Armus, L., Bendo, G., et al. 2003, PASP, 115, 928

Kennicutt, R. C., Calzetti, D., Aniano, G., et al. 2012, PASP, in press

Kovács, A., Chapman, S. C., Dowell, C. D., et al. 2006, ApJ, 650, 592

Li, A., \& Draine, B. T. 2001, ApJ, 554, 778

Lisenfeld, U., Israel, F. P., Stil, J. M., \& Sievers, A. 2002, A\&A, 382, 860

Magrini, L., et al. 2011, A\&A, 515, 13

Meixner, M., Galliano, F., Hony, S., et al. 2010, A\&A, 518, 71

Meny, C., Gromov, V., Boudet, N., et al. 2007, A\&A, 468, 171

Moustakas, J., Kennicutt, R. C., Jr., Tremonti, C. A., et al. 2010, ApJS, 190 233

Ott, S. 2010, in ASP Conf. Ser. 434, Astronomical Data Analysis Software and Systems XIX, ed. Y. Mizumoto, K.-I. Morito, \& M. Ohishi (San Francisco, CA: ASP), 39

Pascale, E., Ade, P. A. R., Bock, J. J., et al. 2009, ApJ, 707, 1740

Pilbratt, G. L., Riedinger, J. R., Passvogel, T., et al. 2010, A\&A, 518, L1

Pilyugin, L. S., \& Thuan, T. X. 2005, ApJ, 631, 231

Poglitsch, A., Waelkens, C., Geis, N., et al. 2010, A\&A, 518, L2

Reach, W. T. 1995, ApJ, 451, 188

Roussel, H. 2011, A\&A, in press

Sandstrom, K., Krause, O., Linz, H., et al. 2010, A\&A, 518, L59

Schlegel, D. J., Finkbeiner, D. P., \& Davis, M. 1998, ApJ, 500, 525

Skibba, R., Engelbracht, C. W., Dale, D., et al. 2011, ApJ, 738, 89

Smith, J. D. T., Draine, B. T., Dale, D. A., et al. 2007, ApJ, 656, 770

Walter, F., Cannon, J. M., Roussel, Hé., et al. 2007, ApJ, 661, 102

Walter, F., Sandstrom, K., Aniano, G., et al. 2011, ApJ, 726, 11

Weingartner, J. C., \& Draine, B. T. 2001, ApJ, 548, 296

Wright, E. L., Mather, J. C., Bennett, C. L., et al. 1999, ApJ, 381, 200 